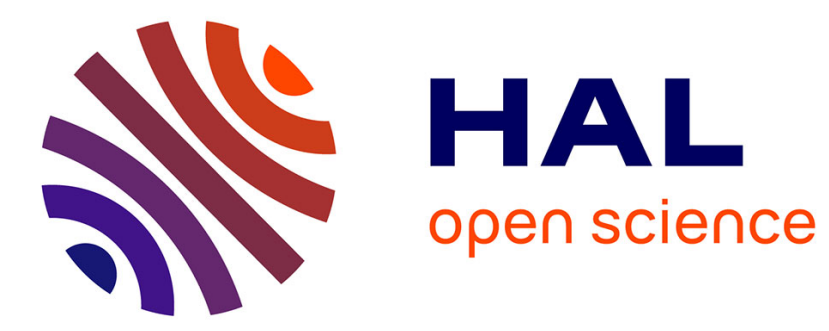

\title{
Model based estimation of ellipsoidal object using artificial electric sense.
}

Sylvain Lanneau, Frédéric Boyer, Vincent Lebastard, Stéphane Bazeille

\section{To cite this version:}

Sylvain Lanneau, Frédéric Boyer, Vincent Lebastard, Stéphane Bazeille. Model based estimation of ellipsoidal object using artificial electric sense.. The International Journal of Robotics Research, 2017, 10.1177/0278364917709942 . hal-01550624

\section{HAL Id: hal-01550624 \\ https://hal.science/hal-01550624}

Submitted on 29 Jun 2017

HAL is a multi-disciplinary open access archive for the deposit and dissemination of scientific research documents, whether they are published or not. The documents may come from teaching and research institutions in France or abroad, or from public or private research centers.
L'archive ouverte pluridisciplinaire $\mathbf{H A L}$, est destinée au dépôt et à la diffusion de documents scientifiques de niveau recherche, publiés ou non, émanant des établissements d'enseignement et de recherche français ou étrangers, des laboratoires publics ou privés. 


\title{
Model based estimation of ellipsoidal object using artificial electric sense
}

Journal Title

$\mathrm{XX}(\mathrm{X}): 1-31$

(C)The Author(s) 2015

Reprints and permission:

sagepub.co.uk/journalsPermissions.nav

DOI: $10.1177 /$ ToBeAssigned

www.sagepub.com/

(SAGE

\section{Sylvain Lanneau ${ }^{1}$, Frédéric Boyer ${ }^{1}$, Vincent Lebastard ${ }^{1}$, Stéphane Bazeille ${ }^{1}$}

\begin{abstract}
In this article we address the issue of shape estimation using electric sense inspired by the active electric fish. These fish can perceive their environment by measuring the perturbations in a self-generated electric field caused by nearby objects. The approach proceeded in three stages. Firstly the object was detected and its electric properties (insulator or conductor) identified. Secondly, the object was localized using the MUSIC (MUltiple Slgnal Classification) algorithm, which was originally developed to localize a radio wave emitter using a network of antennas. Thirdly, the shape estimation relied on the concept of generalized polarization tensor (GPT), which enabled modeling the electric response of an object polarized by an ambient electric field. We describe the implementation of the approach through numerous experiments. The system was able to estimate shape with an average error of $16 \%$, and opened the way toward further improvements. In particular, self aligning the sensor with the ellipsoid through a reactive feedback makes the shape estimation errors drop to $10 \%$.
\end{abstract}

\section{Keywords}

Bio-inspired robotics, artificial electric-sense, shape estimation, object localization

${ }^{1}$ Ecole des Mines de Nantes, France

Corresponding author:

Sylvain Lanneau, Ecole des Mines de Nantes, 4, rue Alfred Kastler B.P. 20722 - F-44307 NANTES Cedex 3, France.

Email: sylvain.lanneau@mines-nantes.fr 


\section{Introduction}

Underwater sensing in turbid conditions remains a challenging issue in robotics today. In muddy waters cluttered with obstacles, neither cameras nor sonar are of use to a robot in performing the usual tasks of reactive navigation, path planning or shape recognition Lane (2012). In recent years, the bio-robotics community have been inspired by the weakly electric-fish, which are able to sense their nearby surroundings by measuring the perturbations of a self-generated electric field caused by nearby obstacles Lissmann and Machin (1958). Also termed the active electric fish, these animals, such as the elephant fish Gnathonemus Petersii, have evolved a specific electric organ located in their tail, that is polarized with respect to the rest of their body, and produces a dipolar electric field in their immediate environment. If an object enters this field, the field is perturbed and these perturbations are measured by a dense array of electro-receptors covering the fish's skin von der Emde and Schwarz (2002). This sixth sense enables electric fish to navigate cluttered environments and muddy waters with great agility, escape from predators, seek prey, and travel long distances. Recent biological experiments showed that electric fish can also perform complex cognitive tasks such as object recognition von der Emde (2006); Hofmann et al. (2013). In these experiments, after a few days of training, the electric fish were able, using only their electric sense, to distinguish between objects which varied in size, electric nature and shape. This is remarkable, since the electric images projected onto the fish skin are fundamentally blurry and a priori difficult to interpret Caputi et al. (1998). Moreover the electric response of the polarized objects show that their position and their intrinsic geometry (shape, size) are intricately linked to each other in the measure of the transcutaneous currents flowing across the skin Rasnow (1996).

Artificial electric sense has been developed in recent years to address several issues in underwater robotics including reactive navigation Boyer et al. (2013), object electrolocation Solberg et al. (2008); Lebastard et al. (2013) and underwater docking Boyer et al. (2015), but shape recognition has been addressed only recently Bai et al. (2015). The approach uses a sensor, the SensorPod, first introduced in Bai et al. (2012). It is an axis-symmetric insulating shell, featuring a set of small electrodes, arrayed on its surface, with a top-bottom, left-right, and front-rear symmetry. The electrodes located at the extremities of the sensor are set under voltage, which generates an electric field in the sensor's environment. Perturbations in this electric field are measured through the voltage recorded between left-right symmetric pairs of floating ground electrodes. Using this device, the authors addressed the issue of shape, size, orientation, and position estimation of ellipsoidal inert (insulating or conductive) objects placed within the sensor's range in its equatorial plane. Exploiting the symmetries of the sensor with respect to the object, the object is first aligned and centered with respect to the SensorPod. Secondly, using maps built beforehand through simulation, its aspect ratio is determined. Finally, the lateral distance and the object's size are estimated with an equivalent sphere model and a gaussian process regression (GPR) model Rasmussen and Williams (2006).

Prepared using sagej.cls 
In the present article, we develop another object estimation strategy based on the physical models recently presented in Boyer et al. (2012) and Ammari, Boulier, Garnier and Wang (2014) and apply it experimentally to another artificial electric sensor based on the measurement of currents Servagent et al. (2013). As in Bai et al. (2015), the objects are three-dimensional inert ellipsoids made of an insulating or a conductive material. Beside object estimation, the main aim of the article is three-fold. First, we want to understand the main physical phenomena involved in shape recognition with electric sense. Second, by experimenting recent models in the field, we want to assess their validity and infer the effects that should be modelled in future for improving shape estimation. Finally, and to a less extent, the approach also contributes to assess a hierarchical strategy conjectured by biologists von der Emde (2006). According to this approach, we first detected the presence of an object while identifying its electric nature (insulator or conductor). Secondly, the object was localized, and thirdly its shape was estimated by processing the electric currents measured along a given trajectory. To this end, we used mathematical tools derived from Ammari, Boulier, Garnier and Wang (2014) as applied to small simulated objects in Lanneau et al. (2016). After a MUSIC-based localization, the shape estimation begins with a least-square estimation of the components of the dipolar leading order generalized polarization tensor (GPT) of the object Ammari, Boulier, Garnier and Wang (2014). This GPT is then inverted with respect to the shape parameters (volume and aspect ratio). Several extensions were required to adapt this approach to our experimental context. In particular, to overcome the low signal/noise ratio of our sensor, the real objects needed to be large and close enough to the sensor. In these conditions, the localization strategy needed to be adapted to cope with the non negligible quadripolar response of the object.

The article is structured as follows. Section 2 presents the experimental and theoretical context of the article as well as a simple detection algorithm which enables the detection of object and the identification of its electric property (conductor or insulator) before its localization and shape estimation. In section 3, the MUSIC localization algorithm is presented and verified empirically on large objects. Section 4 presents the shape estimation algorithm, which is briefly illustrated in simulation, and then evaluated experimentally. The experimental results are presented and discussed in section 5. Based on this discussion, an improved shape estimation is presented in the same section. The article ends with some concluding remarks and perspectives in section 6.

\section{Experimental and theoretical context, object detection}

\subsection{Bio-inspired sensor}

The sensor we used in the article is termed a slender probe for its high aspect ratio (length/diameter). It is composed of four metal rings named macro-electrodes, and denoted $\mathcal{E}_{i=1,2,3,4}$. These macro-electrodes are connected by cylindrical insulating plastic parts as illustrated in Figure 1 left. Each macro-electrode can be polarized, i.e., set to a controlled electric potential $u$, with respect to the others which are all grounded. This polarization of the probe is performed with a wave generator operating at $22 \mathrm{kHz}$.

Prepared using sagej.cls 

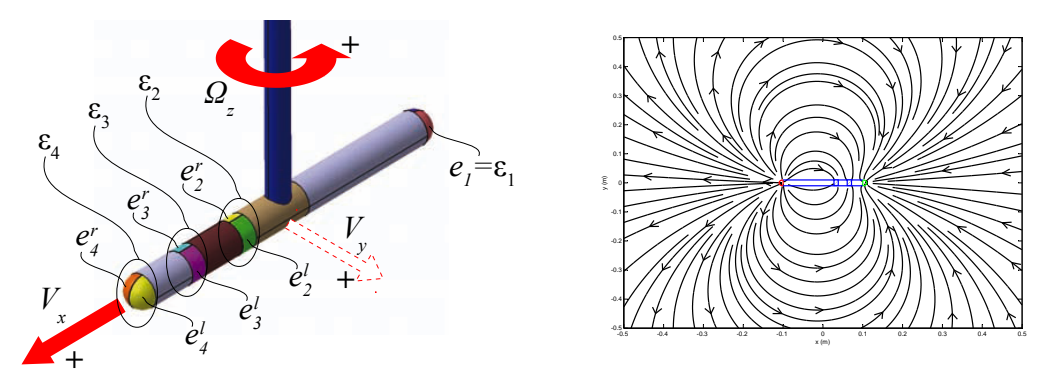

Figure 1. Left: Schematics of the probe with its 4 macro-electrodes divided in left-right measurement electrodes. Right: Planar view of the basal dipolar electric field produced by the probe when the tail electrode $\mathcal{E}_{1}$ is polarized with respect to all the other (grounded) electrodes.

This generates an electrical field, with current flowing through the conductive medium surrounding the probe. When there is no object nearby the probe, this electric field is termed 'the basal field', and denoted $\mathbf{E}_{0}$. Such a basal field is illustrated in figure 1-right, when the tail macro-electrode $\mathcal{E}_{1}$ is polarized. In this case the field clearly exhibits a dipolar shape mimicking that of the fish. Each macro-electrode $\mathcal{E}_{i}$ is divided into two symmetric left and right sub-electrodes denoted $e_{i}^{l}$ and $e_{i}^{r}$, with $\mathcal{E}_{i}=e_{i}^{l} \cup e_{i}^{r}$ as shown in figure 1-left. These sub-electrodes are electrically isolated, so the flux of current penetrating them can be measured independently and gathered in the vector of electric currents $I=\left(I_{1}^{l}, I_{1}^{r}, I_{2}^{l}, I_{2}^{r}, \ldots I_{n}^{l}, I_{n}^{r}\right)^{T}$, with $n$ the total number of rings $(n=4$ for the probe in figure 1) and $I_{i}^{l}$ (respectively $I_{i}^{r}$ ), the total current flowing through the left (respectively, right) half of $\mathcal{E}_{i}$. From these measured currents it is relevant for perception and navigation purposes to use an alternative set of measurements named 'axial' and 'lateral' currents, which can be easily deduced (online) from the $2 n$ components of $I$ through the simple combinations

$$
i=1,2 . . n: I_{\mathrm{ax}, \mathrm{i}}=\left(I_{i}^{l}+I_{i}^{r}\right) / 2, I_{\mathrm{lat}, \mathrm{i}}=\left(I_{\mathrm{i}}^{l}-I_{\mathrm{i}}^{r}\right) / 2 .
$$

Though (1) theoretically holds true for all $\mathcal{E}_{i}$, in practice, our acquisition system is such that for a given polarized macro-electrode $\mathcal{E}_{i}$, only the $I_{\mathrm{lat}, \mathrm{j}}$ and $I_{\mathrm{ax}, \mathrm{j}}$ of the three other grounded electrodes (i.e., such that $j \neq i$ ) can be measured. However, as the electric currents are conserved, the current $I_{\mathrm{ax}, \mathrm{i}}$ flowing across the polarized electrode $\mathcal{E}_{i}$, can be easily computed as the opposite of the sum of the axial currents penetrating the three others. Nevertheless, this reconstruction does not hold for the lateral currents, since in this case the conservation law involves the currents flowing across the left and right sub-electrodes of the same $\mathcal{E}_{i}$ Boyer et al. (2012). In this practical context, for the $\mathcal{E}_{1}$-polarization of figure 1-right, we will consider the two vectors of currents $I_{\mathrm{ax}}=$ $\left(I_{\mathrm{ax}, 1}, I_{\mathrm{ax}, 2}, \ldots I_{\mathrm{ax}, \mathrm{n}}\right)^{T}$ and $I_{\mathrm{lat}}=\left(I_{\mathrm{lat}, 2}, I_{\mathrm{lat}, 3}, \ldots I_{\mathrm{lat}, \mathrm{n}}\right)^{T}$. Moreover, such a pair of vectors can be measured for the $n$ possible polarizations of the sensor. In particular, for the sensor in figure 1 , we can take $U^{T}=(u, 0,0,0),(0, u, 0,0),(0,0, u, 0)$ and $(0,0,0, u)$ and build a set of $2 n=8$ such vectors. When there is no object in the scene surrounding the probe, the bilateral symmetry imposes $I_{\text {lat }}=0$ for any polarization, 
while $I_{\mathrm{ax}}$ is entirely modelled by the following matrix-equation

$$
I_{\mathrm{ax}}^{(0)}=C^{(0)} U,
$$

where $U$ is the $n \times 1$ vector of potentials imposed by the voltage generator and $C^{(0)}$ is a $n \times n$ matrix modelling the basal (indexed (0)) conductivity of the surroundings. This matrix, which only depends on the sensor's geometry and the medium's conductivity $\gamma$, must be determined at the outset either by numerical computation or in situ through a preliminary calibration phase but may then be used as a constant. When an object appears in the sensor's surroundings, the vectors of measured currents $I_{\mathrm{ax}}$ and $I_{\text {lat }}$ are defined for any polarization, by

$$
I_{\mathrm{ax}}=I_{\mathrm{ax}}^{(0)}+\delta I_{\mathrm{ax}}, I_{\mathrm{lat}}=\delta I_{\mathrm{lat}},
$$

where $\delta I_{\mathrm{ax}}$ and $\delta I_{\text {lat }}$ are some perturbative vector components imaging the presence of the object. These measurements, obtained for all polarizations, will be used throughout the article as the support of the information exploited by our shape estimation strategy. Their model is introduced in the next section.

\subsection{Model of the sensor measurements}

The model of currents represents the direct electric problem which consists of determining the electrical currents flowing through the electrodes in any given scene for any polarization. In contrast, the inverse problem consists of reconstructing the scene (its geometry and physics) from the knowledge of the polarization and the measurements of the probe. We have two models at our disposal for the direct problem. Both are fully described in Boyer et al. (2012), but we will briefly present them in this section. The first model, the boundaries elements method (BEM), provides a numerical reference solution of the direct problem, which can be used instead of the measured currents given by experiments. The second model, the analytical model, describes the measured currents with simple explicit mathematical expressions deduced from a reduction process described in Boyer et al. (2012). The methods used for localization and shape estimation being based upon this analytical model, we will recall it in subsection 2.2.2. But we must first define more precisely the scenes in which our sensor is immersed.

2.2.1 Definition of the scenes In all of the following, we will consider the 4electrode probe of figure 1 which has a length $l=220 \mathrm{~mm}$ and a diameter $2 R=20 \mathrm{~mm}$. We will assume that: (1) the conductivity $\gamma$ of the surrounding medium (water) is constant over time and space ; (2) there is only one object in the scene surrounding the sensor ; (3) the object is made of an ideal conductor (e.g. metal) or an ideal insulator (e.g. plastic) ; (4) that object has the shape of a prolate ellipsoid, i.e. it is axisymmetric about its major axis, of length $2 a$, which is assumed to be in the equatorial plane of the sensor. The objects tested in the subsequent experiments are shown in table 1 , where $a$ and $b$ denote the half-length of the major and minor axis respectively, $\eta=a / b$ is the aspect ratio (AR) of the ellipsoid, and $V$ its volume. Under these basic assumptions, any scene can be parameterized as indicated in figure 2 .

Prepared using sagej.cls 


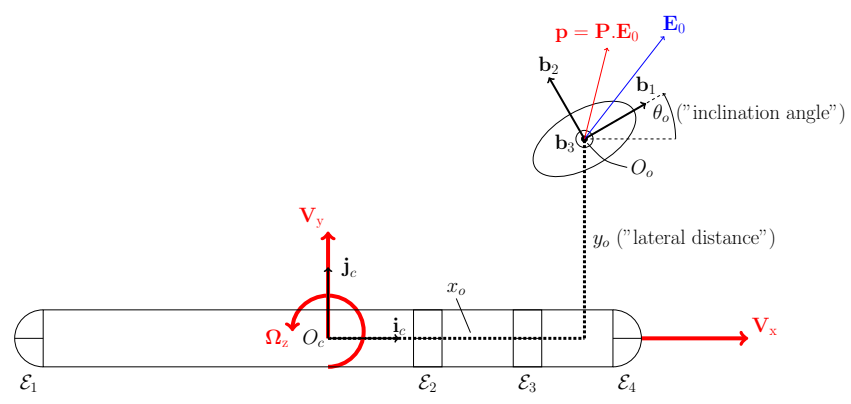

Figure 2. Parametrization of a scene (top view). The coordinates of the geometrical center of the object are denoted $\left(x_{o}, y_{o}\right)$ in the probe's frame $\left(O_{c}, \mathbf{i}_{c}, \mathbf{j}_{c}\right)$. Its orientation relative to the probe's main axis, or inclination angle, is $\theta_{o} . \mathbf{V}_{x}$ and $\mathbf{V}_{y}$ are the longitudinal and lateral vector velocity respectively, and $\boldsymbol{\Omega}_{z}$ is the angular velocity vector about the vertical axis.

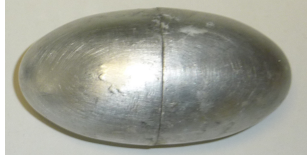

(a) Object 1

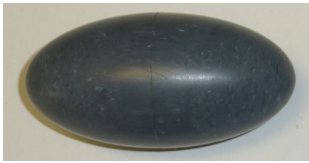

(d) Object 2

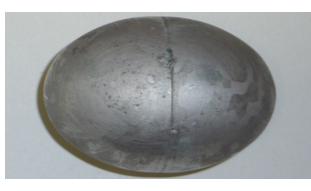

(b) Object 3

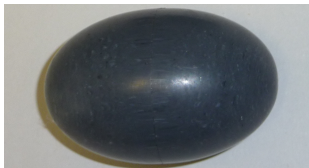

(e) Object 4

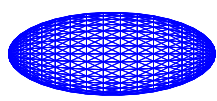

(c) Object 5

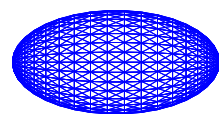

(f) Object 6

Figure 3. Experimental objects ( 1 to 4$)$ and simulated objects ( $5 \& 6)$ (see also table 1$)$.

\begin{tabular}{|c|c|c|c|c|c|c|}
\hline Object number & Type & Material & $a(\mathrm{~cm})$ & $b(\mathrm{~cm})$ & $\eta$ & $V\left(\mathrm{~cm}^{3}\right)$ \\
\hline Object 1 & Experimental & Conductive & 3.3 & 1.6 & 2 & 35.39 \\
\hline Object 2 & Experimental & Insulating & 3.3 & 1.6 & 2 & 35.39 \\
\hline Object 3 & Experimental & Conductive & 2.7 & 1.8 & 1.5 & 36.64 \\
\hline Object 4 & Experimental & Insulating & 2.7 & 1.8 & 1.5 & 36.64 \\
\hline Object 5 & Simulated & Conductive & 1 & 0.4 & 2.5 & 0.67 \\
\hline Object 6 & Simulated & Insulating & 1 & 0.5 & 2 & 1.05 \\
\hline
\end{tabular}

Table 1. Characteristics of the objects experimentally and numerically tested in this paper: material, semi-major axis $a$, semi-minor axis $b$, aspect ratio $\eta$ and volume $V$.

2.2.2 Analytical model of the sensor response in a scene The derivation of the analytical model of Boyer et al. (2012) is based on the following simplifications which 
will be assumed to be fulfilled by our scenes, at least in a first step. Firstly, the probe has a slender shape. Secondly, each object is small enough to consider $\mathbf{E}_{0}$ as being uniform on the object's domain. In practice, this is justified when the object is sufficiently far from the probe $\left(y_{o} \geq 3 R\right.$ in figure 2$)$ and when the object's size is at most of the order of the sensor's radius $(\max (a, b) \leq R)$. In these conditions, the following linear relationships between the vector of input voltages $U \in \mathbb{R}^{4}$, and the vectors of measured currents $I_{\mathrm{ax}} \in \mathbb{R}^{4}$ and $I_{\text {lat }} \in \mathbb{R}^{3}$ may be derived

$$
I_{\mathrm{ax}}=\left(C^{(0)}+\frac{1}{4 \pi \gamma} C^{(0)} G P^{c} G^{\mathrm{T}} C^{(0)}\right) U, I_{\mathrm{lat}}=\left(\frac{1}{4 \pi} P_{\perp} H P^{c} G^{\mathrm{T}} C^{(0)}\right) U,
$$

in which the $4 \times 4$ and $3 \times 4$ real matrices $\delta C=(4 \pi \gamma)^{-1} C^{(0)} G P^{c} G^{\mathrm{T}} C^{(0)}$ and $(4 \pi)^{-1} P_{\perp} H P^{c} G^{\mathrm{T}} C^{(0)}$ define the axial and lateral scene conductance variation matrix caused by the presence of the object. It was shown in Boyer et al. (2012) that $G$ and $H$ are two transmission matrices of dimension $4 \times 3$ and $3 \times 3$ respectively. They depend nonlinearly on the object's coordinates in the sensor frame through the expressions

$$
G=\left(\begin{array}{ccc}
\frac{x_{o}-x_{1}}{r_{1}^{3}} & \frac{y_{o}}{r_{1}^{3}} & 0 \\
\vdots & \vdots & \vdots \\
\frac{x_{o}-x_{4}}{r_{4}^{3}} & \frac{y_{o}}{r_{4}^{3}} & 0
\end{array}\right), H=\left(\begin{array}{ccc}
\frac{3 y_{o}\left(x_{o}-x_{2}\right)}{r_{2}^{5}} & \frac{2\left(y_{o}\right)^{2}-\left(x_{o}-x_{2}\right)^{2}}{r_{2}^{5}} & 0 \\
\frac{3 y_{o}\left(x_{o}-x_{3}\right)}{r_{3}^{5}} & \frac{2\left(y_{o}\right)^{2}-\left(x_{o}-x_{3}\right)^{2}}{r_{3}^{5}} & 0 \\
\frac{3 y_{o}\left(x_{o}-x_{4}\right)}{r_{4}^{5}} & \frac{2\left(y_{o}\right)^{2}-\left(x_{o}-x_{4}\right)^{2}}{r_{4}^{5}} & 0
\end{array}\right)
$$

with $r_{k=1 \ldots n}=\sqrt{\left(x_{o}-x_{k}\right)^{2}+\left(y_{o}\right)^{2}}, x_{k}$ the axial coordinates of $\mathcal{E}_{k}$ in the sensor frame, and where the zero third columns indicate that the scene is planar. Note that in (4), the location of the object with respect to the sensor is only contained in $G$ and $H$. On the other hand, the matrix $P^{c}$ is a polarization tensor that entirely models the response of the object. As such, it only depends on the object's material properties and orientation as detailed in the next subsection. As with $P^{c}, P_{\perp}=\operatorname{diag}_{i=2, \ldots 4}\left(p_{\perp, i}\right)$ in the analytical expression (4) of $I_{\text {lat }}$ is a polarization matrix, here of $3 \times 3$ dimension, which depends only on the sensor's geometry, modeling how the electrodes are laterally polarized by the reflected field. In practice, a numerical value of the matrix $\delta C=$ $(4 \pi \gamma)^{-1} C^{(0)} G P^{c} G^{\mathrm{T}} C^{(0)}$ for any given scene can be easily measured online column by column. Each macro-electrode is successively set to $1 \mathrm{~V}$, the three others to $0 \mathrm{~V}$, and $\delta I_{\mathrm{ax}}$ is measured. The same process can be used to construct the extra-diagonal terms of $(4 \pi)^{-1} P_{\perp} H P^{c} G^{\mathrm{T}} C^{(0)}$. In the following, the model of axial currents along with the matrix $\delta C$ will be used for the localization, while the model of $I_{\text {lat }}$ will assist object detection and shape estimation.

2.2.3 A model for the object's electrical response According to Ammari and Kang (2007), the electric response of an ellipsoidal object to a uniform electric field $\mathbf{E}_{0}$ is characterized by its first order generalized polarization tensor $\mathbf{P}$, or Polya-Szego tensor, which, in the object's principal basis $\left(O_{o}, \mathbf{b}_{1}, \mathbf{b}_{2}, \mathbf{b}_{3}\right)$, can be expressed as a $3 \times 3$ diagonal matrix denoted $P$. This tensor acts on $\mathbf{E}_{0}$, to give the electric field reflected by the object according to the usual relation $\mathbf{E}_{1}(\mathbf{r})=\left(\mathbf{P} . \mathbf{E}_{0}\right) \cdot \mathbf{r} /\left(4 \pi \gamma\|\mathbf{r}\|^{3}\right)$, with $\mathbf{r}$ the position vector with respect to the object's center, and $\mathbf{p}=\mathbf{P}$. $\mathbf{E}_{0}$, the dipole induced on the object by its polarization by $\mathbf{E}_{0}$ (see figure 2). For ellipsoids with high conductivity 
(conductive) or low conductivity (insulating) with respect to water, $P$ depends only on the object's volume $V$ and the aspect ratio $\eta=a / b$ through the expression*:

$$
P=\left(\begin{array}{ccc}
\lambda_{1} & 0 & 0 \\
0 & \lambda_{2} & 0 \\
0 & 0 & \lambda_{2}
\end{array}\right)=V\left(\begin{array}{ccc}
f(\eta) & 0 & 0 \\
0 & g(\eta) & 0 \\
0 & 0 & g(\eta)
\end{array}\right)
$$

where $(f, g)=(1 / A, 1 / B)$, or $(f, g)=(1 /(A-1), 1 /(B-1))$ depending whether upon the object is conductive or insulating, while the two functions $A$ and $B$, are elliptic integrals defined as

$$
\left\{\begin{array}{l}
A(\eta)=\eta^{-2} \int_{1}^{+\infty} \frac{1}{t^{2}\left(t^{2}-1+\eta^{-2}\right)} d t \\
B(\eta)=\eta^{-2} \int_{1}^{+\infty} \frac{1}{\left(t^{2}-1+\eta^{-2}\right)^{2}} d t
\end{array}\right.
$$

The ellipsoid major axis is rotated by an angle $\theta_{o}$ about the vertical axis relatively to the sensor main axis. Thus, in the sensor frame, the matrix of the components of $\mathbf{P}$, denoted $P^{c}$ is defined as

$$
P^{c}=R\left(\theta_{o}\right) P R\left(\theta_{o}\right)^{\mathrm{T}} \Rightarrow\left(\begin{array}{c}
p_{11}^{c} \\
p_{12}^{c} \\
p_{22}^{c}
\end{array}\right)=\left(\begin{array}{c}
\lambda_{1} \cos ^{2}\left(\theta_{o}\right)+\lambda_{2} \sin ^{2}\left(\theta_{o}\right) \\
\left(\lambda_{1}-\lambda_{2}\right) \cos \left(\theta_{o}\right) \sin \left(\theta_{o}\right) \\
\lambda_{1} \sin ^{2}\left(\theta_{o}\right)+\lambda_{2} \cos ^{2}\left(\theta_{o}\right)
\end{array}\right),
$$

where $p_{i j}^{c}$ denotes the $(i, j)$ component of $P^{c}$, while $R\left(\theta_{o}\right)$ is the $3 \times 3$ rotation matrix of angle $\theta_{o}$ about the vertical axis. Note that (8) naturally appears in the expressions (4) of the sensor response to the presence of a polarized object.

\subsection{Preliminary basic experiment}

To introduce the experimental conditions of all the tests reported in the article, we will here consider a simple preliminary experiment named 'fly-by' test in Bai et al. (2012). This simple test allows us to assess the accuracy of our models and to reproduce some concepts that were recently introduced in the biological literature Hofmann et al. (2013). These concepts will be used later (section 3.3) to assist the localization process.

2.3.1 Experimental setup The probe described in section 2.1 is immersed in a cubical tank of side $1 \mathrm{~m}$ and attached to one end of a rigid epoxy fiber tube. The other end of this tube is attached to a Cartesian robot, or 'gantry', fixed on the top of the tank. The gantry allows the center probe's position to be controlled along the horizontal $x$ and $y$ axes, and to rotate about the vertical $z$ axis (see figure 4). Motion control is performed with a DSpace ${ }^{\circledR}$ system running a custom program. For a complete

* Note that beyond a sphere and an ellipsoid, the exact expression of this tensor has not been derived. 


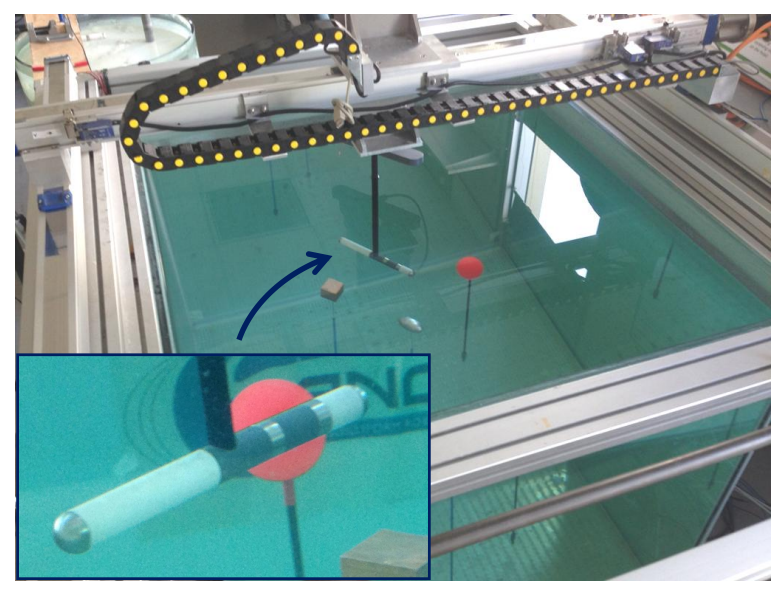

Figure 4. The gantry above the water tank. The probe's motion through a scene composed of objects lying in the probe's equatorial plane is controlled from above.

description of the robot (electronics schematics, noise level, positioning precision, sensitivity to temperature) we refer the reader to Servagent et al. (2013). The various scenes described in section 2.2, are composed by placing objects of varying shape, size and material at various locations in the tank.

2.3.2 Preliminary 'fly-by' test We first consider the probe when it moves forward in straight line along its main axis, to sweep past an insulating ellipsoid (object 2 in table 1). A constant polarization vector $U=(1,0,0,0)^{T}$ is set, while the axial and lateral currents $\delta I_{\mathrm{ax}}$ and $I_{\text {lat }}$ are measured throughout the trajectory. The object is located at a lateral distance of $80 \mathrm{~mm}$ and rotated by $30^{\circ}$ with respect to the probe's axis. The water conductivity is $0.04 \mathrm{~S} / \mathrm{m}$. Figures 5 and 6 show the plots of $I_{\text {lat }}$ and $\delta I_{\text {ax }}$ measured experimentally (with a $1 \mathrm{~mm}$ spatial resolution), and as computed by the BEM and analytical models, as a function of the longitudinal position of the probe's center with respect to the object's center. Note that according to (3), to compute $\delta I_{\mathrm{ax}}$, we need to remove the effect of the insulating tank's walls. To that end, the axial currents are measured along the same path that the sensor took during the fly by, but without the object, and then subtracted to those measured when the object is present. This example is illustrative of many similar tests performed with the objects described in table 1. Note that, except for some amplitude differences, the analytical and BEM models faithfully describe the measured currents.

2.3.3 Peak position and t-images Biologists have recently studied temporal images (t-images) in electric fish, which are similar to the fly-by plots of lateral currents illustrated in figure 5. In their conclusions, they conjectured that while moving past an object, fish could detect the maximum of the transcutaneous currents flowing across one receptor, or peak-position (PP), to infer the object position along the rostrocaudal axis Hofmann et al. (2013). In the following, we will exploit the same idea by remarking that when the lateral current of a given electrode reaches its maximum, one can infer 


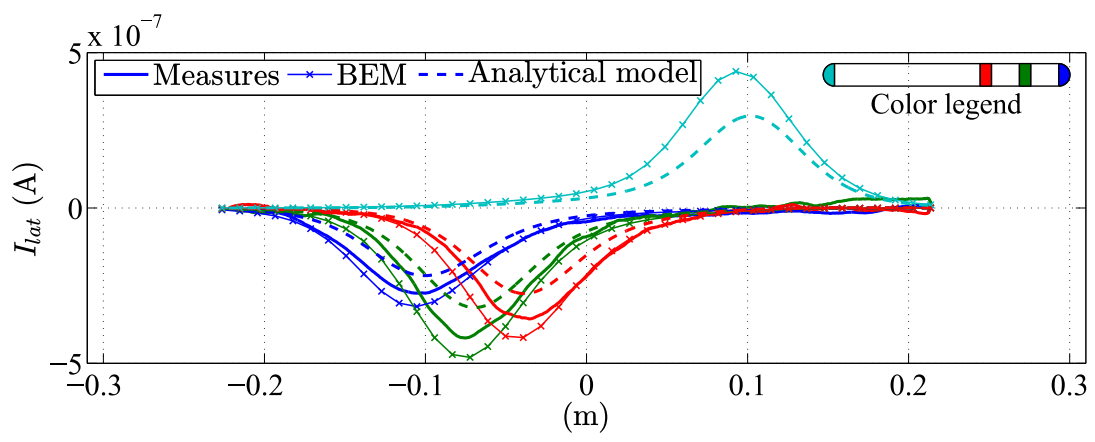

Figure 5. Currents $I_{\text {lat }}$ for the probe motion of Figure 2 (fly-by test in conditions detailed in the text), versus the longitudinal position of the probe's center w.r.t. the object's center. Solid lines: measured currents. Solid lines with crosses: BEM currents. Dashed lines: currents calculated with the analytical model.

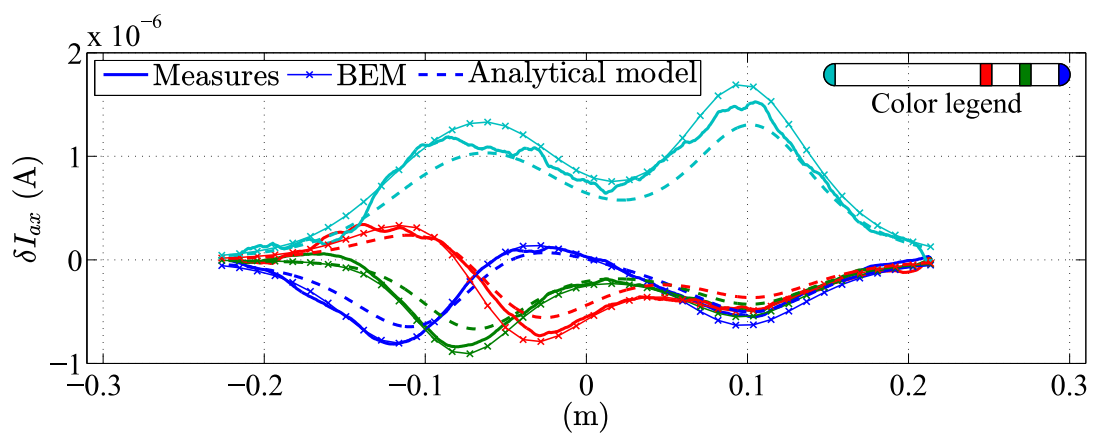

Figure 6. Same as Figure 5, but for the 4 axial currents of $\delta I_{\mathrm{ax}}$.

that a perturbative object faces this electrode. The plots of figure 5 will be termed timages, and a maximum of a lateral current will be termed a peak-position (PP). These concepts along with the analysis of fly-by plots will be used to assist localization in section 3.3.2 and to identify the object material as this is detailed in the next section.

\subsection{Detection algorithm}

In Boyer et al. (2013), an analysis of the fly-by plots with the polarization of figure 1-b showed that $\delta I_{\mathrm{ax}}$ can be used to infer whether a perturbative object is an insulator or a conductor, while $\delta I_{\text {lat }}$ can be used to infer whether it is on the left or the right side of the probe. In detail, noting $\Sigma I_{\text {lat }}=\sum_{i=2}^{4} I_{\text {lat }, \mathrm{i}}$, and $\Sigma \delta I_{\mathrm{ax}}=\sum_{i=2}^{4} \delta I_{\mathrm{ax}, \mathrm{i}}=-\delta I_{\mathrm{ax}, 1}$, we have the following rules on axial currents:

1. If $\Sigma \delta I_{\mathrm{ax}}=0$, there is no object in the scene;

2. If $\Sigma \delta I_{\mathrm{ax}}>0$, then the object is a conductor;

3. If $\Sigma \delta I_{\mathrm{ax}}<0$, then the object is an insulator.

For lateral currents we have: 
1. If $\Sigma I_{\text {lat }}=0$, there is no object or there is one facing the head electrode;

2. If $\Sigma I_{\text {lat }}>0$, a conductor is on the left or an insulator is on the right;

3. If $\Sigma I_{\text {lat }}<0$, an insulator is on the left or a conductor is on the right.

These simple rules can be easily combined and implemented in a preliminary 'detection algorithm' before object localization. This algorithm informs the global shape estimation strategy, by identifying the presence of an object in the scene, its electric nature (insulator/conductor) and on which side of the probe it is located. In practice, the zero value of currents must be replaced by a threshold fixed by the noise level of the sensor Servagent et al. (2013).

\section{Object localization using MUSIC}

After detection, the next step before shape estimation consists of the localization of the object independently of its intrinsic properties and orientation. To that end, we use the MUSIC algorithm which has been applied in simulation to a dense array of receptors in Ammari, Boulier, Garnier and Wang (2014). This section details the implementation of the same algorithm, but in our experimental setting which deploys a physical sensor with a much sparser array of receptors.

\subsection{Principle of the algorithm}

MUSIC is a model-based sensor array signal processing algorithm Krim and Viberg (1996). It was developed in the 1980s to estimate the direction of arrival of electromagnetic or sound waves by a passive antenna network Schmidt (1986). In the new millennium, Devaney showed that the algorithm's principle could be extended to a network of active antennas, in order to localize scatterers in an homogeneous medium Devaney (2000). By sequentially emitting a polarization wave from each antenna of the network, and by measuring the polarized scatterers' responses using the other receivers, a data matrix named multi-static response matrix, or MSRM, was recorded. The matrices which relate $U$ to $\delta I_{\text {ax }}$ and $I_{\text {lat }}$ in (4), may potentially define such a MSR matrix, at least in theory. However, since in practise we cannot measure the lateral current on the emitting electrode, the MSR matrix will be defined as the $4 \times 4$ conductance variation matrix $\delta C$, which can be measured on line as described in section 2.2.2. As developed in Devaney (2000), the analytic expression of $\delta C$ in (4) defines a set of vector fields, called steering vectors corresponding to the Green function vectors of the background medium. In our particular case, these steering vectors are simply the two 4-dimensional vector fields in the $(x, y)$ plane of the scene

$$
\left\{\begin{array}{l}
S_{x}(x, y)=C^{(0)}\left[\begin{array}{llll}
\frac{x-x_{1}}{r_{1}^{3}} & \frac{x-x_{2}}{r_{2}^{3}} & \frac{x-x_{3}}{r_{3}^{3}} & \frac{x-x_{4}}{r_{4}^{3}}
\end{array}\right]^{\mathrm{T}}, \\
S_{y}(x, y)=C^{(0)}\left[\begin{array}{llll}
\frac{y}{r_{1}^{3}} & \frac{y}{r_{2}^{3}} & \frac{y}{r_{3}^{3}} & \frac{y}{r_{4}^{3}}
\end{array}\right]^{\mathrm{T}},
\end{array}\right.
$$

where $\left(x_{i}, y_{i}\right), i=1,2,3,4$ denote the coordinates of $\mathcal{E}_{i}$ (its center), in the sensor frame $\left(O_{c}, \mathbf{i}_{c}, \mathbf{j}_{c}\right)$, while $r_{i}$ is here defined as $r_{i}=\sqrt{\left(x-x_{i}\right)^{2}+y^{2}}$. The steering vector fields have two important properties. Firstly, they do not depend on the intrinsic properties of the object (shape, material, size) nor on its orientation. Secondly, when 
evaluated in the object's location, i.e., when $(x, y)=\left(x_{o}, y_{o}\right)$, they lie in the signal subspace $\mathcal{S}$, or equivalently, their projection onto the noise subspace $\mathcal{N}$, obtained with the operator $P_{\mathcal{N}}$, is zero, and the following imaging function

$$
F(x, y)=\frac{1}{\left\|P_{\mathcal{N}} S_{x}(x, y)\right\|+\left\|P_{\mathcal{N}} S_{y}(x, y)\right\|},
$$

is infinite Devaney (2000). Defining a grid attached to the sensor frame in which each node is a candidate object location, and using the fact that the projection matrix $P_{\mathcal{N}}$ of (10) can be directly deduced from the 2 singular vectors of lowest singular values of the measured $\delta C$, one can state the following localization algorithm:

1. measure the MSR matrix $\delta C$ by activating the polarized macro-electrodes in sequence, as described in section 2.2.2;

2. compute the SVD of the matrix $\delta C$;

3. select the singular vectors associated with the lowest singular values in order to form a basis of the noise subspace $\mathcal{N}$;

4. in each node of a grid attached to the sensor frame, evaluate the steering vectors (9), and compute the imaging function $F$ of (10);

5. once all nodes have been treated, select the node for which $F$ is the highest, and consider this node to represent the object's location.

This algorithm represents our variant of the MUSIC algorithm and its implementation is described below. As it is based on the object's dipolar response model, this algorithm can be considered to be a localizer of the center of a dipole independent of its orientation and intensity.

\subsection{Simulation of the MUSIC algorithm}

The MUSIC localization algorithm was applied to a broad set of simulated small objects with the BEM currents as inputs in Lanneau et al. (2016). Figure 7 shows one representative example of the numerical results obtained for the small insulating ellipsoidal object (simulated object number 6 in table 2). In all cases, MUSIC underestimated the lateral distance between the sensor and the object. This probably arises from the analytical model outlined in section 2.2, which estimates the electrical response of an object by its leading order dipolar component. In reality, such responses are an infinite multipolar expansion Landau and Lifshitz (1984); Ammari, Garnier, Kang, Lim and Yu (2014). In particular, the non-uniformity of the basal electric field $\mathbf{E}_{0}$ causes further components of the multipolar response of the object that we neglect in our present model. Intuitively, because $\mathbf{E}_{0}$ strongly decreases with lateral sensor-object distance, MUSIC tends to overweight the part of the object closest to the probe as this is illustrated in figure 8-top, which shows how the location error made by MUSIC increases with object volume. Another source of further multipolar components is introduced by the asphericity of the object. This is illustrated by figure 8-bottom, which shows how the localization error (here along the rostrocaudal axis) increases with the aspect ratio for a big ellipsoid of fixed volume. Finally, figure 9 shows a map of the localization error for a small test conductive sphere of diameter equal to that of the sensor, the error (in \%), being related to sensor length. This map shows the theoretical 

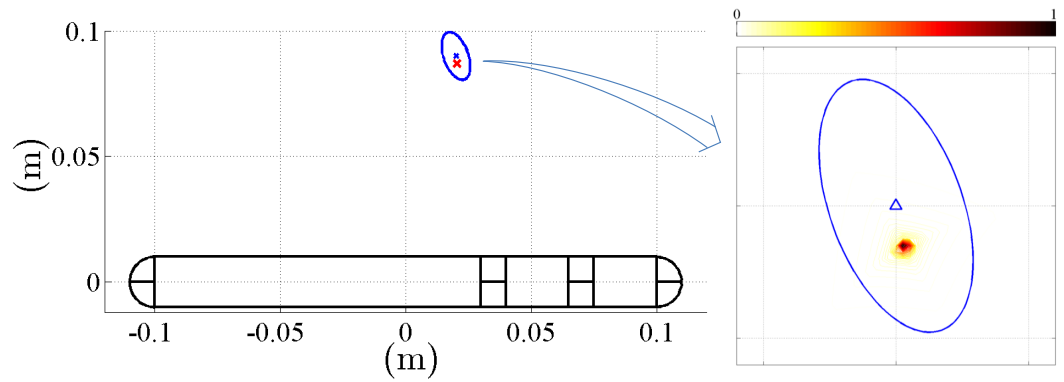

Figure 7. Representative example of the application of MUSIC to the small insulating object of table 2, with BEM currents as input. On the left, the red cross represents the estimated localization. On the right, focused view of the object with the peak of the (normalized) imaging function.

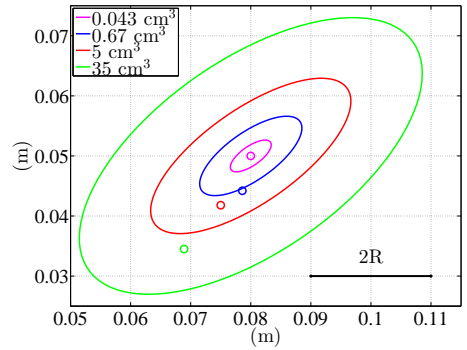

(a)

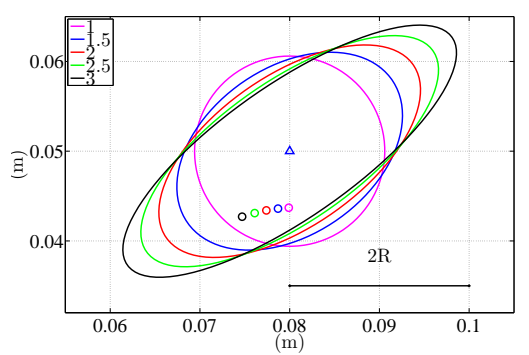

(c)

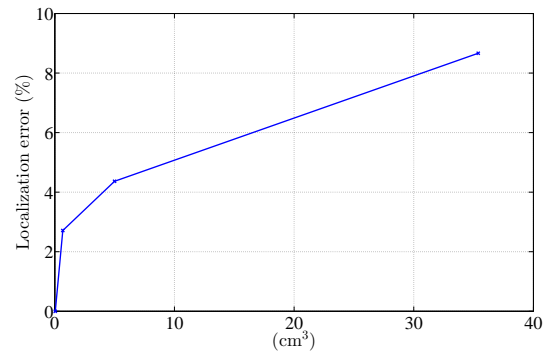

(b)

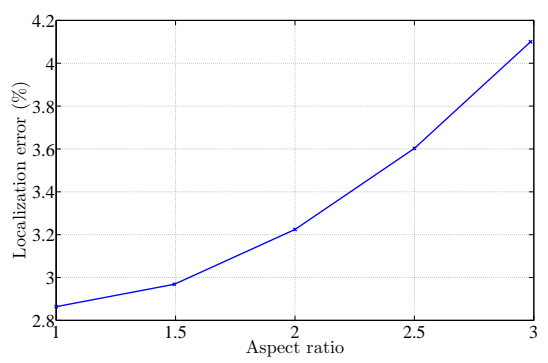

(d)

Figure 8. (Top left) MUSIC localizations for several object's volumes with the BEM (fixed aspect ratio: 2.5). (Top right) Localization error as function of the object's volume. (Bottom left) MUSIC localizations for several object's aspect ratio with the BEM (fixed volume: $5 \mathrm{~cm}^{3}$ ). (Bottom right) Localization error as function of the object's aspect ratio.

efficiency of the algorithm even for a sparse array of receptors aligned on the same 


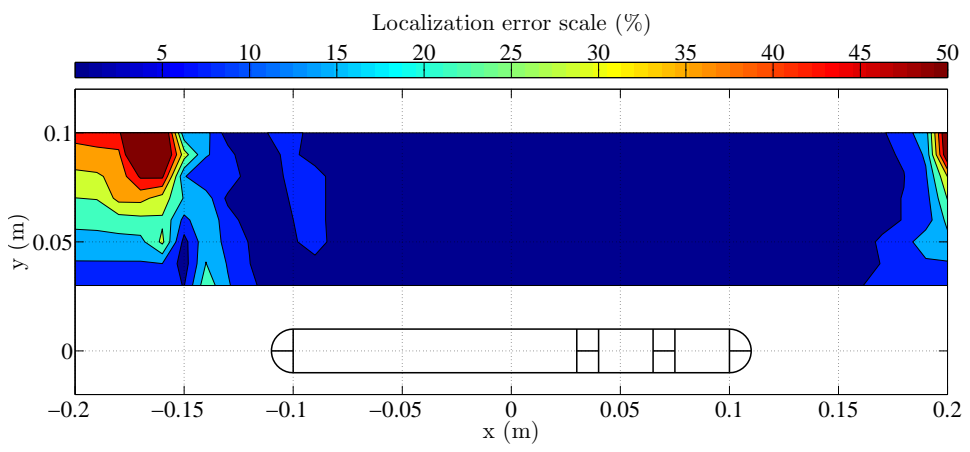

Figure 9. MUSIC error map for a small (same diameter as that of the probe) conductive sphere in BEM.

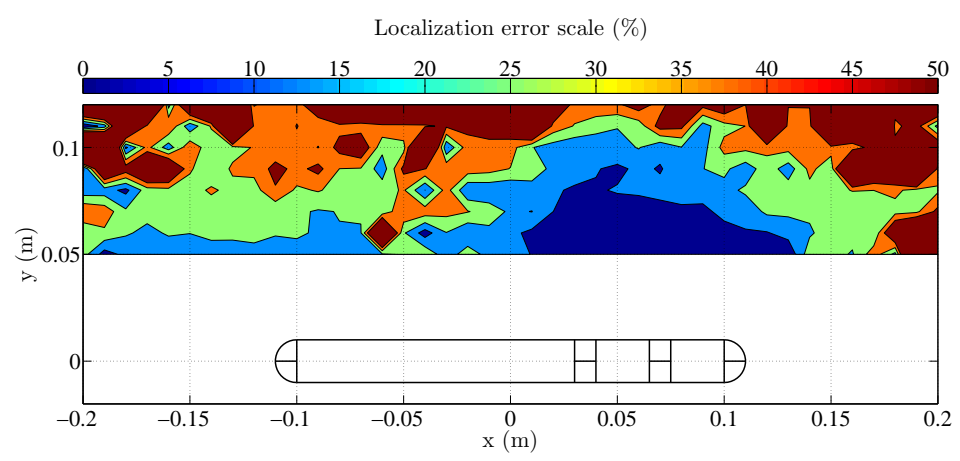

Figure 10. MUSIC error map for a conductive ellipsoid (object 1 in table 1).

straight line ${ }^{\dagger}$. Nevertheless, this arrangement restricts the localization to the region that laterally bounds the probe and does not work in its front or rear sides.

\subsection{Experimental application of the MUSIC algorithm}

3.3.1 From small to large objects In the experimental conditions presented in section 2.3, the signal-to-noise ratio (SNR) when testing the small objects of Lanneau et al. (2016) dramatically restricted the range of detection, which falls to only a few centimeters. To increase the range of our experimental measurements, we will now consider the large objects $1,2,3,4$ of table 1 , even if they do not respect a priori the hypothesis of our analytical model, and see if we can overcome this limit. As we shall see, this will require a new localization strategy for large objects to be derived.

3.3.2 New localization strategy for large objects Although large objects can be detected by the probe, applying the MUSIC algorithm to them considerably decreases

${ }^{\dagger}$ In optimal conditions, MUSIC is applied to a network arranged in a circle surrounding the scene 


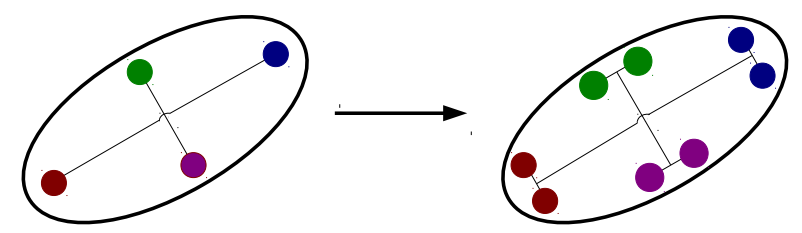

Figure 11. From the dipolar to the quadripolar response of an object. When the object is large compared with the space-variations of the basal field, or when the sensor is sufficiently close to the object, the dipolar response along each of its principal axes, changes to a quadripolar response. Each monopole of the dipoles aligned with the principal axis (green, blue, purple and red colored lumped electric charges in the figure) splits into a dipole centered on the corresponding monopole. In these conditions, MUSIC localizes the center of each of these sub-dipoles.

the precision of the localization. This is illustrated in figure 10, which displays the map of the localization error, calculated in the same conditions as those of figure 9, but with ellipsoid 1 of table 1, i.e. with a large physical object, instead of a small simulated one. As we discussed in section 3.2, the fall off in MUSIC performance may be explained by the fact that due to the object's size, the basal field is far from uniform over the entire volume, and the objects response reveals further multipolar terms that are not present in the model in section 2.2, nor in the steering vectors in section 3.1. Although pushing the multipolar expansion beyond the leading order contribution of the Polya-Szego tensor is quite involved, especially for a three-dimensional object Ammari, Garnier, Kang, Lim and Yu (2014), one can intuitively approximate the quadripolar response by four dipolar responses, each of them modeling the local response of the four top surfaces of the ellipsoid as schematized in figure 11. Based on this simplified model, we propose to apply MUSIC to each of these sub-dipoles, as we did in section ?? for the single dipolar response of a small object, and to estimate the object's location as the barycenter of location of these 4 dipoles. To recover the conditions of section ?? while performing this strategy, the probe is motion-controlled along a square trajectory as schematized in figure 12, and MUSIC is applied once to each side of this square. To automate the approach and optimize the efficacy of MUSIC, we use the bio-inspired concepts of t-image and peak position introduced in section 2.3.3. To that end, let us first remark that, in figure 10, the experimental resolution of MUSIC for large objects is maximal when the object faces the neck electrode $\mathcal{E}_{3}$. Then, using the conclusions of section 4, the MUSIC algorithm has to be applied along each side of the square at the peak position of $I_{\text {lat }, 3}$ for the tail-polarization of figure 1-b. Practically, that means that the probe must stop at the PP, start the multi-polarization of the probe to compute the MSRM $\delta C$, and this is then fed into the MUSIC algorithm described in section ??. At the edges of the square, the probe is rotated by $\pi / 2$, the rotation being started after the probe has been translated half of its axial length, ensuring that the tail has passed the object. This principle is summarized in the following algorithm which can be performed autonomously: 


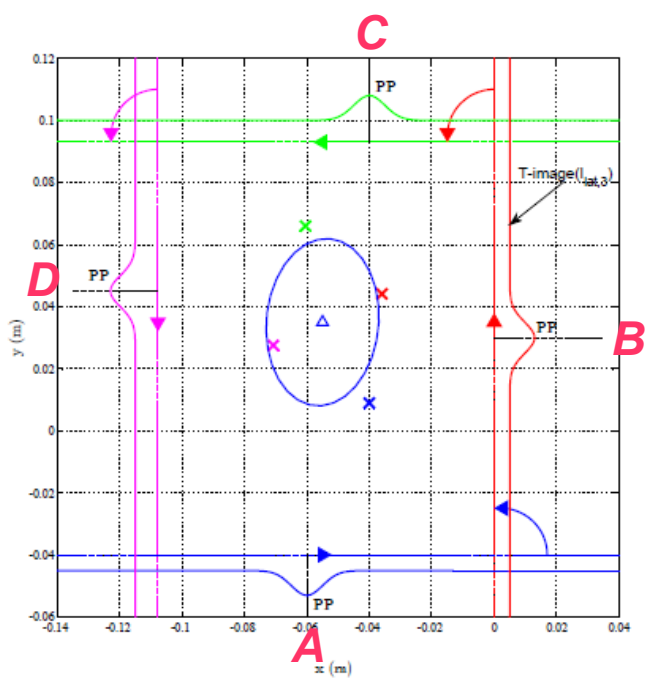

Figure 12. Localization strategy for large objects. The coloured lines represent the rectilinear path followed by the probe. The t-images (of $I_{\mathrm{lat}, 3}$ ) are superimposed along these four paths. When the PP of the t-image is reached, the probe stops and MUSIC computes the object localization, represented by a cross of same colour as the path on which the localization is performed. Taking the barycenter of these four localizations gives an estimation of the object's center localization.

1. while $I_{\mathrm{lat}, 3}$ does not reach a PP, move the probe forward;

2. when $I_{\text {lat }, 3}$ reaches a PP, stop the probe and apply MUSIC;

3. translate the probe half its axial length, and then rotate it by $\pi / 2$;

4. go to step 1 .

Note that in all steps the probe is moved in straight line with $U=(1,0,0,0)^{T}$, except in step 2 where the probe is stopped and the measure of $\delta C$ requires that each of the electrodes be polarized with respect to all of the others. This strategy is summarized in Figure 12, where the t-images of $I_{\mathrm{lat}, 3}$ and their PP are schematized along the sides of the square trajectory. Finally, the barycenter of the four localizations provided by MUSIC is computed and considered as the object's center estimation.

3.3.3 Experimental localization for large objects In this section, we report some representative experimental results provided by the above localization strategy. We considered the large $(\mathrm{AR}=2)$ conductive ellipsoid and the large $(\mathrm{AR}=1.5)$ insulating one (object 1 and 4 of table 1). Each of the two objects was positioned in the tank's center, and the algorithm of section 3.3.2 was applied with the probe starting at a lateral distance of $52 \mathrm{~mm}$ for object 1 , and $63 \mathrm{~mm}$ for object 2 , with object 1 rotated by $-30^{\circ}$, and object 2 rotated by $90^{\circ}$. From these initial conditions, the algorithm, which is applied manually, closes the square trajectory while performing the four MUSIC estimations as soon as $\mathcal{E}_{3}$ faces the object. These four particular scenes (with the 

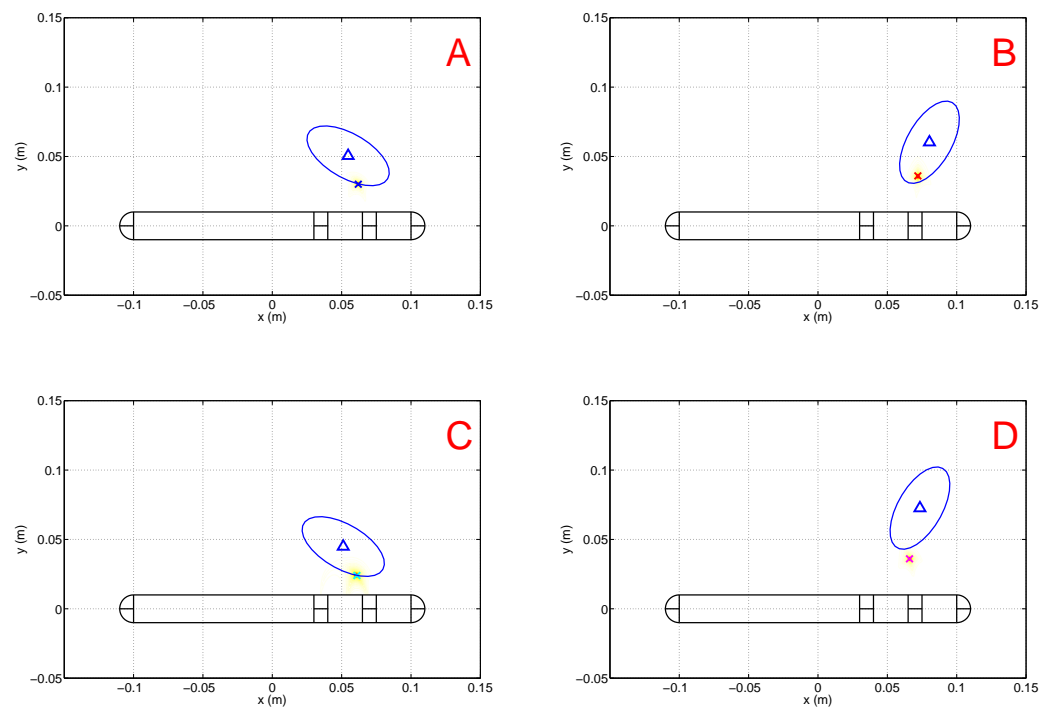

Figure 13. Snapshots of the scene in the probe's frame when MUSIC is performed, i.e. when the PP of $I_{\text {lat }, 3}$ is reached for the object 1 of table 1 . The cross indicates the estimated localization, the triangle stands for the real position of the object's center. The letters $A, B$, C, D correspond to the time at which MUSIC is performed as represented in figure 12 .

corresponding estimated localization provided by MUSIC) are displayed in figure 13 when the strategy is applied to the conductive object (object 1 of table 1). In figure 14, we have drawn the boundaries of the two tested ellipsoids as well as the four localizations estimated by MUSIC along with their barycenter. In the two cases, which are representative of all the cases we tested, the approach estimates the true object's center position with an error of $2.6 \mathrm{~mm}$ and $1.6 \mathrm{~mm}$ respectively. Note also that on the right panel of figure 14, instead of locating the tops of the ellipsoid, MUSIC tends to localize its closest points to the probe. This can be explained by the fact that when the object is inclined, the two sub-dipoles of figure 11 closest to the probe are excited, and their response is mixed to produce that of an apparent dipole between the two ellipsoid axes. Thus, since MUSIC localizes one dipole at a time, this is this apparent dipole which is localized by the algorithm.

\section{Object shape estimation}

Throughout this section, we will assume that the object material (conductor or insulator) and the probe's side on which it is located have been determined using the detection algorithm outlined in section 2.4, while the location of the object in the sensor frame has been estimated using the localization algorithm in section 3.3.2. Shape estimation for ellipsoidal objects requires three parameters to be handled. The first two are strictly related to shape and can be either the semi-axis length $(a, b)$, or 

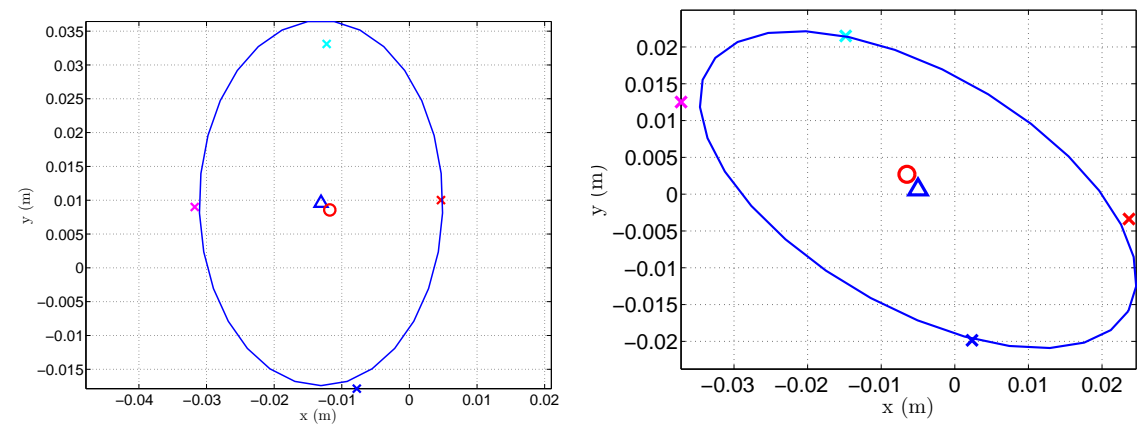

Figure 14. Real boundaries of the ellipsoidal conductive (left) and insulating (right) object with its four localization (indicated by crosses). Each of them is erroneous but their barycenter (circle) gives a good estimation of the object's center (here indicated by a triangle).

equivalently the volume $V$ and the aspect ratio $\eta$. The third parameter is the angle of the ellipsoid $\theta_{o}$ with respect to the probe axis (see figure 2). Though extrinsically related to the object (this is a parameter of the pose), this further angle is estimated in parallel with the shape. Based on this parametrization, the shape estimation algorithm is performed in two steps. First, the components of the polarization tensor in the sensor frame $p_{11}^{c}, p_{12}^{c}$ and $p_{22}^{c}$ are estimated with an optimization algorithm. Secondly, the object's parameters $\left(V, \eta, \theta_{o}\right)$ or $\left(a, b, \theta_{o}\right)$ are computed by inverting the analytical expressions of the components of the tensor (6-8).

4.0.4 Step one: Estimation of the polarization tensor Let us first remark that, according to the model presented in section 2.2, the lateral and axial currents measured on each of the macro-electrodes can be written as linear combinations of the components of $P^{c}$, i.e. for $k=1,2,3,4$ and $m=2,3,4$

$$
\begin{aligned}
& I_{\mathrm{lat}, \mathrm{m}}=\varphi_{1, m} p_{11}^{c}+\varphi_{2, m} p_{12}^{c}+\varphi_{3, m} p_{22}^{c}, \\
& \delta I_{\mathrm{ax}, \mathrm{k}}=\psi_{1, k} p_{11}^{c}+\psi_{2, k} p_{12}^{c}+\psi_{3, k} p_{22}^{c}
\end{aligned}
$$

$\varphi_{1, m}, \varphi_{2, m}, \varphi_{3, m}$, and $\psi_{1, k}, \psi_{2, k}, \psi_{3, k}$ being functions of $\left(x_{o}, y_{o}\right)$ deduced from (4) and (5) and the particular value of $C^{(0)}$. Now, since the position of the object $\left(x_{o}, y_{o}\right)$ is at each instant known in the sensor frame, these functions are computed at sampling instants $t_{i}$ along the controlled trajectory of the probe and paired with the $I_{\text {lat, } \mathrm{m}}\left(t_{i}\right)$ and $\delta I_{\mathrm{ax}, \mathrm{k}}\left(t_{i}\right)$ measurements. Repeating this acquisition process for $i=1,2 \ldots N$ allows the $7 N$ measured currents to be collected along the trajectory to inform the following 

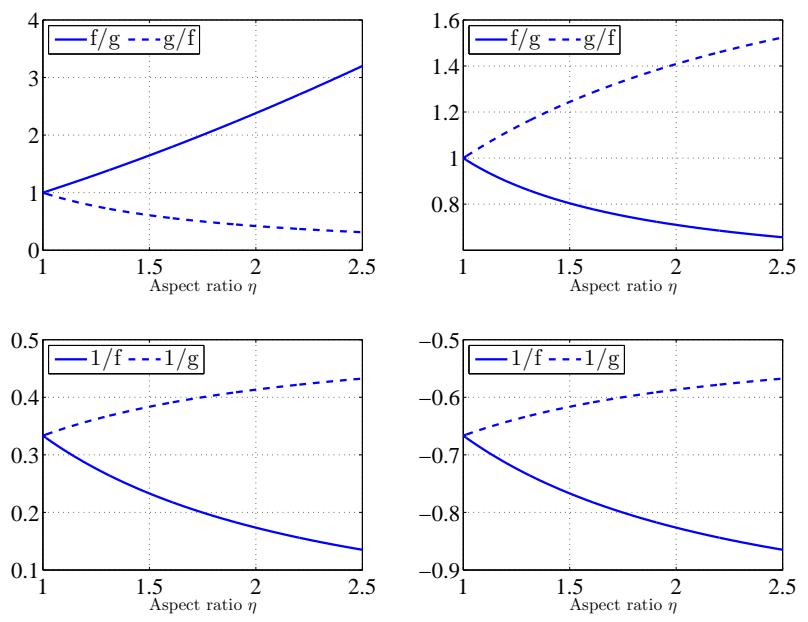

Figure 15. Functions $f / g, g / f, 1 / f$ and $1 / g$ for conductive (left column) and insulating (right column) objects.

system of $7 N$ equations with respect to the three unknowns $p_{11}^{c}, p_{12}^{c}$ and $p_{22}^{c}$

$$
\left\{\begin{aligned}
I_{\mathrm{lat}, \mathrm{m}}\left(t_{1}\right) & =\varphi_{1, m}\left(t_{1}\right) p_{11}^{c}+\varphi_{2, m}\left(t_{1}\right) p_{12}^{c}+\varphi_{3, m}\left(t_{1}\right) p_{22}^{c} \\
\delta I_{\mathrm{ax}, \mathrm{k}}\left(t_{1}\right) & =\psi_{1, k}\left(t_{1}\right) p_{11}^{c}+\psi_{2, k}\left(t_{1}\right) p_{12}^{c}+\psi_{3, k}\left(t_{1}\right) p_{22}^{c} \\
\vdots & \\
I_{\mathrm{lat}, \mathrm{m}}\left(t_{N}\right) & =\varphi_{1, m}\left(t_{N}\right) p_{11}^{c}+\varphi_{2, m}\left(t_{N}\right) p_{12}^{c}+\varphi_{3, m}\left(t_{N}\right) p_{22}^{c} \\
\delta I_{\mathrm{ax}, \mathrm{k}}\left(t_{N}\right) & =\psi_{1, k}\left(t_{N}\right) p_{11}^{c}+\psi_{2, k}\left(t_{N}\right) p_{12}^{c}+\psi_{3, k}\left(t_{N}\right) p_{22}^{c}
\end{aligned}\right.
$$

where $k$ and $m$ run from 1 to 4 and 2 to 4 respectively. On a straight line trajectory, the angle $\theta_{o}$ is constant, as are the components of $P^{c}$. Thus (12) is a linear system of $7 N$ equations in the three unknown components of $P^{c}$ that we can solve with the least squares method. After this first step, we have only to recover the geometric parameters of the object to determine the object's shape.

4.0.5 Step two: Shape parameters estimation To recover the object geometry from the polarization tensor in the sensor frame, we invert the analytical model (8) of section 2.2. This is practically done by remarking that adding and subtracting the first and third line of (8) gives the equivalent alternative system of equations:

$$
\left(\begin{array}{c}
p_{11}^{c}+p_{22}^{c} \\
p_{11}^{c}-p_{22}^{c} \\
2 p_{12}^{c}
\end{array}\right)=\left(\begin{array}{c}
\lambda_{1}+\lambda_{2} \\
\left(\lambda_{1}-\lambda_{2}\right) \cos \left(2 \theta_{o}\right) \\
\left(\lambda_{1}-\lambda_{2}\right) \sin \left(2 \theta_{o}\right)
\end{array}\right) .
$$


Considering the two last lines of this system for $\theta_{o}$ yields:

$$
\left\{\begin{array}{l}
\text { If } p_{12}^{c}=p_{11}^{c}-p_{22}^{c}=0, \text { the object is a sphere and } \theta_{o} \text { is arbitrary. } \\
\text { Else, the object is ellipsoidal and } \theta_{o}=\frac{1}{2} \operatorname{ATAN} 2\left(2 p_{12}^{c}, p_{11}^{c}-p_{22}^{c}\right) .
\end{array}\right.
$$

Moreover, adding the square of the two last lines of (13), and combining the result with its first line, gives for $\lambda_{1}$ and $\lambda_{2}$

$$
\left\{\begin{array}{l}
\lambda_{1}=\frac{1}{2}\left[\left(p_{11}^{c}+p_{22}^{c}\right) \pm \sqrt{\left(p_{11}^{c}-p_{22}^{c}\right)^{2}+4\left(p_{12}^{c}\right)^{2}}\right], \\
\lambda_{2}=\frac{1}{2}\left[\left(p_{11}^{c}+p_{22}^{c}\right) \mp \sqrt{\left(p_{11}^{c}-p_{22}^{c}\right)^{2}+4\left(p_{12}^{c}\right)^{2}}\right],
\end{array}\right.
$$

where the upper and lower signs of \pm and $\mp$ correspond to the cases of a conductor and an insulator respectively. Once the inclination angle $\theta_{o}$ and the intrinsic polarization tensor $P=\operatorname{diag}\left(\lambda_{1}, \lambda_{2}, \lambda_{2}\right)$ are known, the geometry of the ellipsoid may be inferred. To this end, we exploit the model in (6), which shows that the ratio $\lambda_{1} / \lambda_{2}$ depends only on the aspect ratio $\eta$, through the function $f / g$ whose graph is plotted in figure 15 for conductive and insulating objects. In both cases, these functions have only one solution over the range $[1,2.5]$ and $\eta$ can be estimated unambiguously by simply reading the plots of figure 15. Finally, as a third step, still using (6), $V$ can be estimated using $V=\lambda_{1} / f(\eta)$.

\subsection{Numerical shape estimation}

In all the following, $e_{s}=\left[\left((a-\hat{a})^{2}+(b-\hat{b})^{2}\right) /\left(a^{2}+b^{2}\right)\right]^{1 / 2}$ with $(\hat{a}, \hat{b})$ the estimated shape, denotes the relative error (in \%) on the estimated shape vector, and $e_{\theta}=\left|\theta_{o}-\hat{\theta}_{o}\right|, \hat{\theta}_{o}$ being the estimated inclination angle, is the absolute error (in degrees) on the inclination angle. We have shown in Lanneau et al. (2016) that the above shape estimation approach works well on simulated BEM measurements carried out on small objects. Figure 16 shows the estimated shape of small objects 5 (figure 16-left) and 6 (figure 16-right) (see also table 1), at a lateral distance of $50 \mathrm{~mm}$ and $90 \mathrm{~mm}$ and an inclination angle of $35^{\circ}$ and $110^{\circ}$ with respect to the probe's axis. For the conductive object, we found $e_{s}=3.8 \%$ and $e_{\theta}=0.9^{\circ}$ while for the insulating object, we obtained values of $e_{s}=10.5 \%$ and $e_{\theta}=2.8^{\circ}$.

\subsection{Experimental shape estimation}

We assess the same approach this time for the four large objects of table 1 in our experimental test-bed. The shape estimation algorithm will first be applied to objects localized with the gantry proprioception (i.e., by using the angular encoders of the gantry when the probe slighthy touches the object). In a second step we will assess the robustness of shape estimation to the localization errors. In contrast to the algorithm used in the simulation-based context of Lanneau et al. (2016), average filtering with a window of 31 samples (for a total sample amount of 420) was first applied to the input measurements in order to reduce noise. The standard least square algorithm of Lanneau et al. (2016) has also been replaced by its weighted version Ljung (1999),

Prepared using sagej.cls 

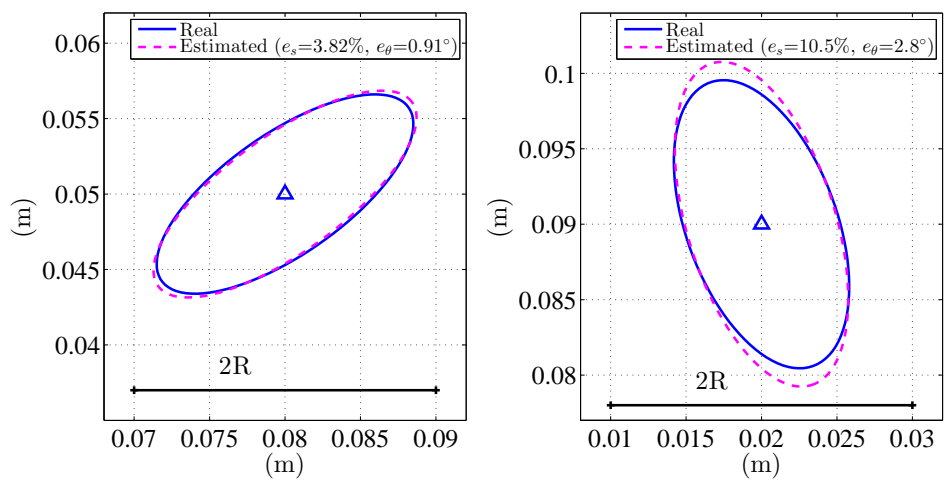

Figure 16. Estimated shapes of the conductive (left) and insulating (right) ellipsoid (objects 5 and 6 in table 1), with no localization error and using the BEM simulator as a direct electric model.
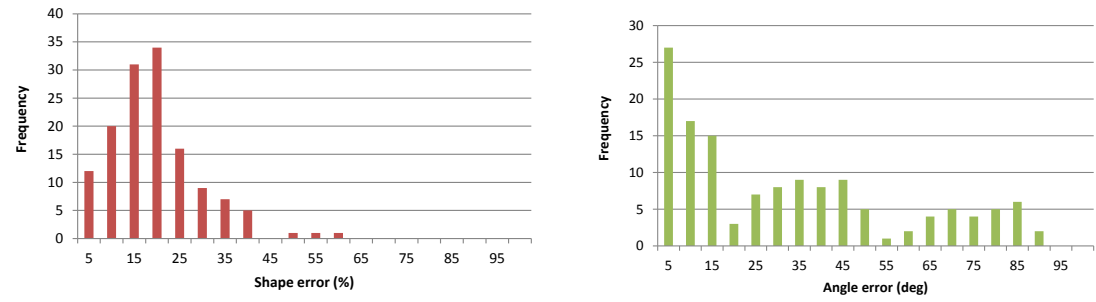

Figure 17. Shape error and angle error distribution over the 137 experiments of table 2.

where the weights of the quadratic residual are defined by the norm $\left\|\mathbf{E}_{0}\right\|$, computed at the corresponding estimated localizations of the object in the sensor's frame. This gives more weight to the measurements in (12) which represent the conditions in which the object is most illuminated by the sensor.

4.2.1 Evaluation of shape estimation results with a priori knowledge of localization The tested objects were placed in our tank, close to its center, with five different orientations ranging from $-30^{\circ}$ to $90^{\circ}$ (in steps of $30^{\circ}$ ). All localizations and orientations were measured in a preliminary step using the proprioception of our motion-controlled gantry. Once the pose of the object had been measured, all the electric currents were recorded along a straight line path of $2 l=440 \mathrm{~mm}$ length, whose lateral distance ranged from $50 \mathrm{~mm}$ to $110 \mathrm{~mm}$ in step of $10 \mathrm{~mm}$ (see figure 2). This varying lateral distance allowed assessment of the range of our shape estimation. The processing of the data was performed off line in Matlab, on a desktop equipped with an Intel core i7 processor, running at $2.8 \mathrm{Ghz}$, with $8 \mathrm{~Gb}$ of RAM. The processing times ranged from 2.02 to 2.40 s for each of the 137 experiments presented in table 2 . 


\begin{tabular}{|c|c|c|c|c|c|c|c|c|c|c|c|}
\hline & & \multicolumn{10}{|c|}{ Table A } \\
\hline & \multirow[b]{2}{*}{$\begin{array}{l}\text { Lat. dist. } \\
(\mathrm{mm})\end{array}$} & \multicolumn{2}{|l|}{-30} & \multicolumn{2}{|l|}{0} & \multicolumn{2}{|c|}{30} & \multicolumn{2}{|c|}{60} & \multicolumn{2}{|l|}{90} \\
\hline & & $\begin{array}{c}\text { Shape } \\
\text { error (\%) }\end{array}$ & $\begin{array}{l}\text { Angle } \\
\text { error }\left(^{\circ}\right)\end{array}$ & \begin{tabular}{|c|} 
Shape \\
error (\%)
\end{tabular} & $\begin{array}{c}\text { Angle } \\
\text { error }\left({ }^{\circ}\right)\end{array}$ & \begin{tabular}{|c|} 
Shape \\
error (\%)
\end{tabular} & $\begin{array}{c}\text { Angle } \\
\text { error }\left({ }^{\circ}\right)\end{array}$ & $\begin{array}{c}\text { Shape } \\
\text { error (\%) }\end{array}$ & $\begin{array}{c}\text { Angle } \\
\text { error }\left({ }^{\circ}\right)\end{array}$ & \begin{tabular}{|c|} 
Shape \\
error (\%)
\end{tabular} & $\begin{array}{c}\text { Angle } \\
\text { error }\left(^{\circ}\right)\end{array}$ \\
\hline Conducting & 50 & 18,10 & 9,25 & 9,07 & 2,07 & 4,35 & 2,63 & 35,79 & 9,30 & & . \\
\hline a & 60 & 8,69 & 6,27 & 10,27 II & 0,89 & 6,96 & 3,25 & $\mid 12,05$ & 3,47 & 34,71 & 11,32 \\
\hline 0,033 & 70 & 4,02 & 4,23 & 12,65 I & 0,27 & 9,02 I & 3,29 & 7,89 & 3,15 & 25,28 & 10,06 \\
\hline $\mathrm{b}$ & 80 & 1,98 II & 1,69 & $\mid 16,81$ & 7,03 & 11,66 I & 3,04 & 3,63 I & 1,45 & 15,16 & 8,91 \\
\hline 0,016 & 90 & $2,27 \|$ & 2,12 & $-17,42$ & 8,21 & $=9,92$ & 6,61 & 1,35 | & 3,01 & 16,13 ! & 8,57 \\
\hline Object 1 & 100 & 5,16 | & 3,51 & 16,20 & 9,27 & 23,34 & 4,11 & 3,38 & 4,34 & 19,62 & 10,19 \\
\hline & 110 & $2,62 \|$ & 1,35 & 21,24 & 0,80 & $\quad 37,52$ & 67,58 & 11,69 & 10,60 & $\quad 12,82$ & 12,24 \\
\hline
\end{tabular}

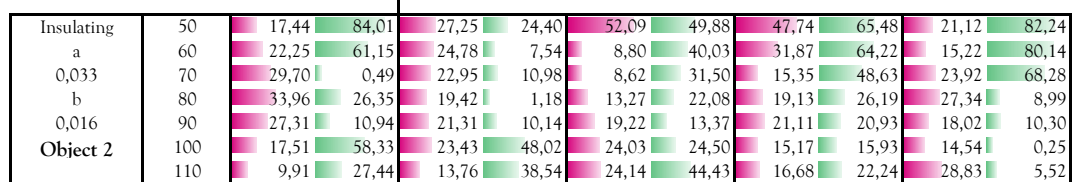

\begin{tabular}{|c|c|c|c|c|c|c|c|c|c|c|c|}
\hline Conducting & 50 & 5,20 & 5,60 & 16,09 & 37,50 & \begin{tabular}{ll|}
14,74 \\
\end{tabular} & 10,16 & 28,66 & $\begin{array}{l}12,58 \\
\end{array}$ & $\begin{array}{l}18,07 \\
\end{array}$ & 31,50 \\
\hline $\mathrm{a}$ & 60 & 11,80 & 2,78 & 17,70 & 21,75 & 14,65 ॥ & 0,35 & 15,75 & & 3,95 & 33,09 \\
\hline 0,027 & 70 & 16,52 & 11,93 & & 86,83 & & & & & 4 & 28,86 \\
\hline $\mathrm{b}$ & 80 & 17,08 & 39,57 & 14, & 75,96 & & 32,69 & 9,49 & 20,26 & 5,61 & 31,30 \\
\hline & 9 & & 36,64 & & & & & & & & 1,26 \\
\hline Object 3 & 100 & 15,06 & 42,68 & & 72,2 & 11,97 & 82,52 & 2,77 & 44,64 & 5,86 & 29,97 \\
\hline & 110 & 31,03 & 36,67 & 9,90 & 65,26 & 12,64 & 88,84 &, 26 & 38,88 & 4,37 마 & 9.5 \\
\hline
\end{tabular}

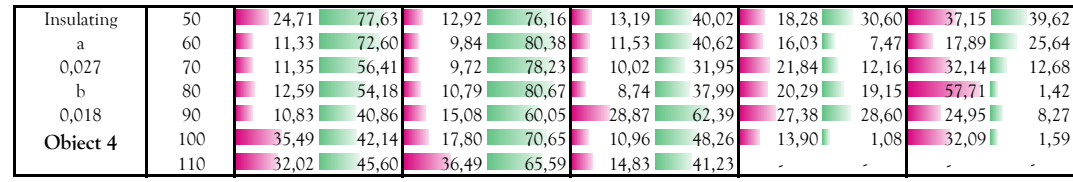

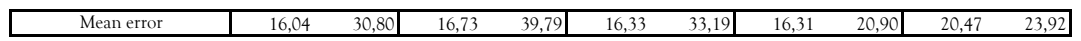

\begin{tabular}{|l|r|r|}
\multicolumn{1}{c}{} & \multicolumn{2}{c}{ Table B } \\
\cline { 2 - 3 } \multicolumn{1}{c|}{} & \multicolumn{1}{c|}{$\begin{array}{c}\text { Shape } \\
\text { error (\%) }\end{array}$} & $\begin{array}{c}\text { Angle } \\
\text { error }\left(^{\circ}\right)\end{array}$ \\
\hline Object 1 & 13,20 & 7,18 \\
Object 2 & 22,21 & 32,99 \\
Object 3 & 12,93 & 37,19 \\
Object 4 & 20,26 & 42,18 \\
\hline
\end{tabular}

Table C

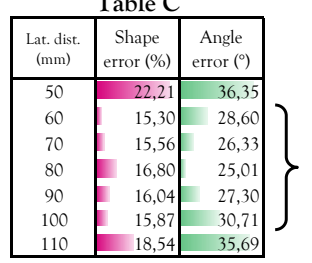

Mean shape: $15,9 \%$

Mean angle: $27,6^{\circ}$

Table D

\begin{tabular}{|c|c|c|c|c|c|c|c|c|}
\cline { 2 - 9 } \multicolumn{1}{c|}{} & \multicolumn{9}{c|}{ Conducting } & \multicolumn{2}{c|}{ Insulating } & \multicolumn{2}{c|}{$\boldsymbol{\eta}=2$} & \multicolumn{2}{c|}{$\boldsymbol{\eta}=1,5$} \\
\cline { 2 - 9 } \multicolumn{1}{c|}{} & Shape $(\%)$ & Angle $\left({ }^{\circ}\right)$ & Shape $(\%)$ & Angle $\left(^{\circ}\right.$ & Shape $(\%)$ & Angle $\left(^{\circ}\right)$ & Shape $(\%)$ & Angle $\left({ }^{\circ}\right)$ \\
\hline Mean error & 11,88 & 21,46 & 19,95 & 33,72 & 16,28 & 16,77 & 15,55 & 38,41 \\
\hline Standard deviation & 6,73 & 24,72 & 9,25 & 25,49 & 8,59 & 20,40 & 9,49 & 26,13 \\
\hline
\end{tabular}

Table 2. Results of the experiments: table A: Errors of shape (pink) and angle (green) for 137 different experiments: 4 objects, 5 angles $\left(-30^{\circ}, 0^{\circ}, 30^{\circ}, 60^{\circ}, 90^{\circ}\right), 7$ distances $(50,60$, $70,80,90,100,110) \mathrm{mm}$. The last line shows the mean of each column. table B: Mean errors of shape and angle for each object. table C: Mean errors of shape and angle for all objects at each distance. table D. Mean errors of shape and angle for conductive objects, insulating objects, and aspect ratio 2 and 1.5 in the distance interval [60 - 100] (100 experiments).

The shape estimation results are reported in the four tables of table 2. In table $2 \mathrm{~A}$, each horizontal block reports the errors computed for each object. In each block, each row records the lateral distance (between the object's center and the probe's straight path), and the columns represent the different inclination angles of the object with 

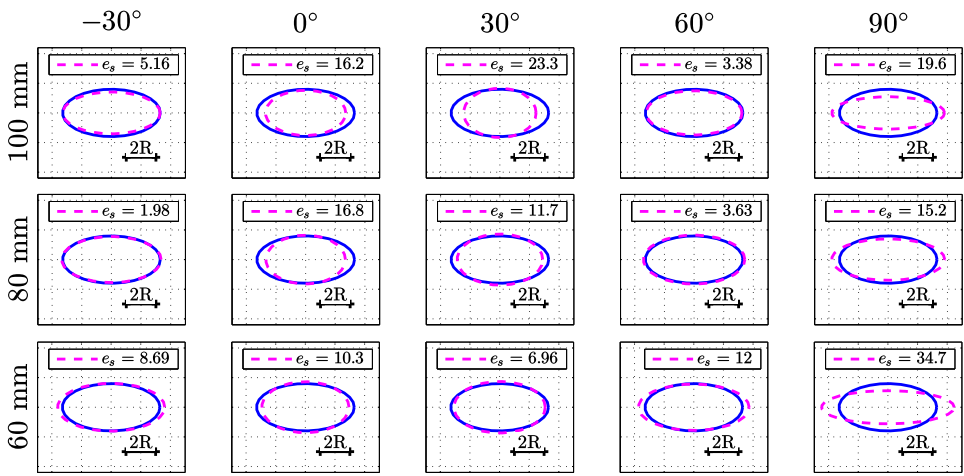

Figure 18. Estimated shapes of a large conductive ellipsoid (object 1 in table 1), for different lateral distances $(60,80,100) \mathrm{mm}$ and inclination angles: $-30^{\circ}, 0^{\circ}, 30^{\circ}, 60^{\circ}, 90^{\circ}$.
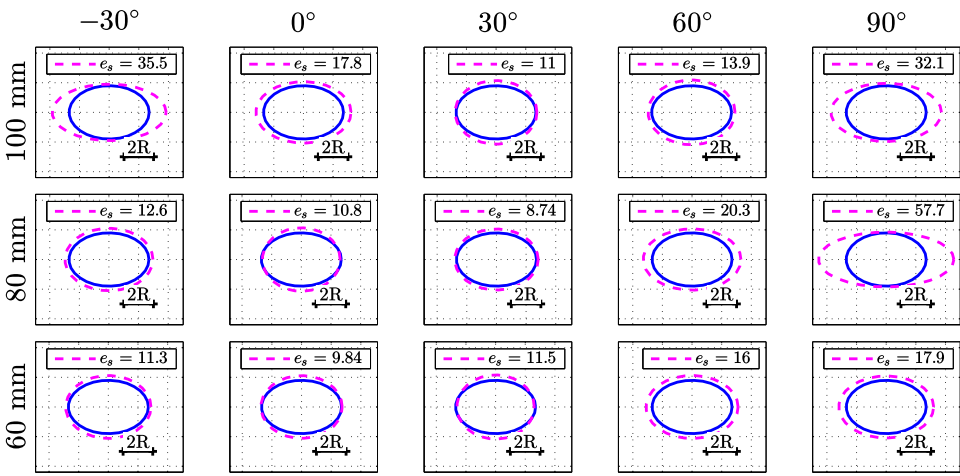

Figure 19. Estimated shapes of a large insulating ellipsoid (object 4 in table 1), for different lateral distances $(60,80,100) \mathrm{mm}$ and inclination angles: $-30^{\circ}, 0^{\circ}, 30^{\circ}, 60^{\circ}, 90^{\circ}$.

respect to the probe path. Note that 3 error values are missing in this table. For the object 1 , at $50 \mathrm{~mm}$ and $90^{\circ}$, since in this case the robot was colliding with the object, and for object 4 , at $110 \mathrm{~mm}, 90^{\circ}$ and $60^{\circ}$, since in this case, the data were too noisy to be exploited. Looking at the results of table $2 \mathrm{~A}$ object by object, one can see that the shape estimation error ranges from $1 \%$ to $57 \%$ with an average of $17 \%$. More than $82 \%$ of the shape errors are lower than $25 \%$, as is apparent in the distribution of shape errors displayed in figure 17-left. The mean of each shape estimation error for each object orientation is presented in the last line of the table $2 \mathrm{~A}$, which shows that these averages are all close to $16 \%$ except when the inclination angle is $90^{\circ}$ where it is more than $20 \%$. Table $2 \mathrm{~A}$ also shows that the angle is not estimated well as confirmed by the wide distribution of angle errors in figure 17-right. Better accuracy was obtained for the first object, but significant errors (up to $90^{\circ}$ in some cases) for the 3 others were obtained, whatever their initial orientation and lateral distance. Remarkably, the poor performance of inclination angle estimation does not much affect our shape estimation results, as confirmed by table $2 \mathrm{~B}$ which shows the mean of the shape and angle errors 
for each object.

In table $2 \mathrm{C}$ we report the shape errors (respectively angle errors) computed as the sum of the shape error for all objects at each distance. This shows that the interval of reliable distances using our method is [60-100] $\mathrm{mm}$. This is easily explained by the fact that the identification approach is based on the model of the dipolar response of the object. This assumption is justified when the object is not too close to the sensor, while increasing the sensor-object distance reduces the signal/noise ratio, and algorithm performance decreases. In this reliable interval, the model of the estimator is more justified and the mean shape error falls to $15.9 \%$. This value quantifies the global performance of the approach, customized for these conditions. Table 2D shows the shape and angle errors obtained with objects of same electric nature (insulator or conductor), and with the same aspect ratio in this optimal range. The mean shape error for the conductors falls to $12 \%$ while that of insulators is $20 \%$. Note that a shape error of $20 \%$ can be quite acceptable as illustrated in figures 18 and 19, where we have drawn the real and estimated shapes of objects 1 and 4 . The shape of conductive objects is globally better estimated than that of insulators. Similarly, the ellipsoids of smaller aspect ratio are better estimated than those with a larger aspect ratio. However, table 2D also shows that the inclination angle of objects with small aspect ratio is difficult to estimate.

4.2.2 Evaluation of the influence of the localization uncertainty on shape estimation To illustrate the influence of localization error on shape estimation we here report some shape estimation results obtained with a conductive ellipsoid positioned at $60 \mathrm{~mm}$ and rotated in $30^{\circ}$-steps from $-30^{\circ}$ to $90^{\circ}$ with respect to the probe main axis. The shape estimation algorithm has been repeated with different errors on the object localization systematically sampled along the polar grid of figure 20-bottomright, centered on the object position. In the following, this object position which is measured using the robot gantry before each experiment is called 'ground truth'. Note that the maximal tested localization error $(6 \mathrm{~mm})$ is quite large compared to the errors reported in section 3.3, which do not exceed $3 \mathrm{~mm}$. The top row of figure 20 shows the estimated ellipses for each dot of the grid in figure 20-bottom-right. Figure 20-bottomleft shows the shape errors averaged along a circle of the polar grid, and denoted $\bar{e}_{s}$, as a function of the localization error $e_{\rho}$. These results show how the accuracy of shape and angle estimation rapidly decreases as localization error increases. In table 3 , the shape error is compared for two objects: (1) with the ground truth localization, (2) with the experimental localization based on the multi-MUSIC strategy of section 5, and (3) with the 50 erroneous localizations of figure 20-bottom-right. The average shape error calculated over the $6 \mathrm{~mm}$ error circle for the conductive object was below $14 \%$, and around $35 \%$ for the insulating object.

\section{Discussion}

In the reliable range for which the shape estimation algorithm has been developed, the overall shape error is around $16 \%$, while it did not exceed $10 \%$ on BEM simulations carried out with small simulated objects Lanneau et al. (2016). The differences between 

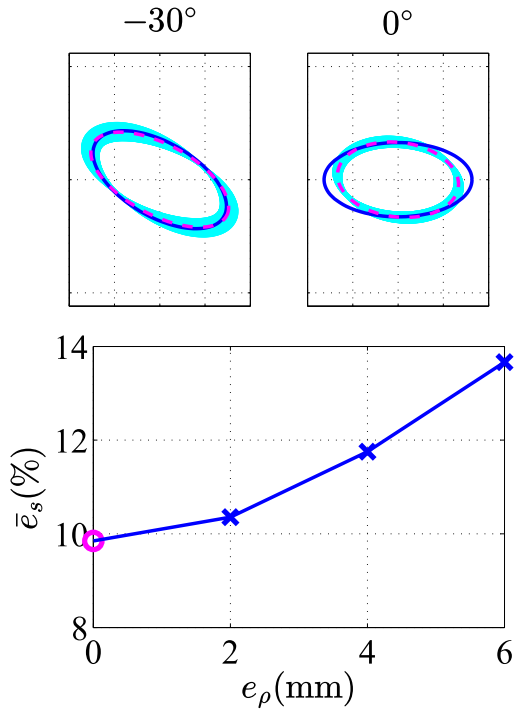

$30^{\circ}$

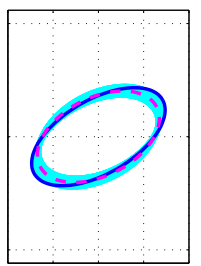

$60^{\circ}$

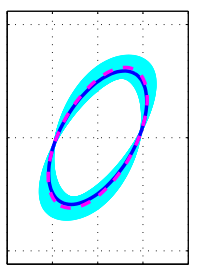

$90^{\circ}$

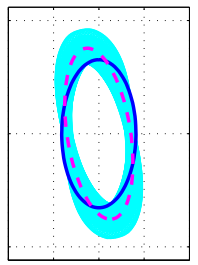

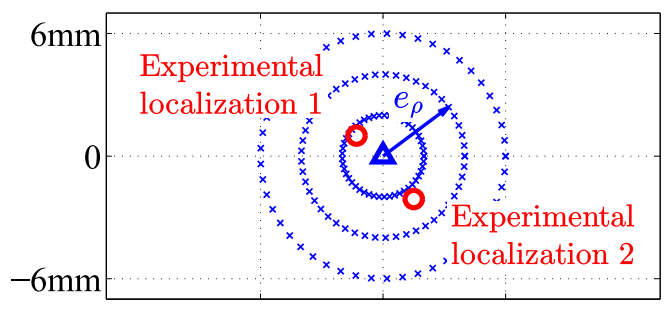

Figure 20. Shape estimation of object 1 in table 1 at $60 \mathrm{~mm}$ for 5 orientations (from left to right: $-30^{\circ}, 0^{\circ}, 30^{\circ}, 60^{\circ}, 90^{\circ}$ ). The additional error $e_{\rho}$ (of the localization) have been introduced as inputs of shape estimation, as illustrated in the polar grid of the bottom right panel. (Top) Estimated ellipsoid for the $3 \times 50$ erroneous localization (pale blue); real shape (blue); estimated shape when the localization is perfect (pink dashed lines). (Bottom left) Mean averaged shape error $\bar{e}_{s}$ over 50 experiments, as a function of the localization error $e_{\rho}$. (Bottom right) Grid of the erroneous localizations centered on the ground truth localization represented by the blue triangle. It is defined by 3 circles of $\{2,4,6\} \mathrm{mm}$ radius and by 50 equidistant angles from $0^{\circ}$ to $360^{\circ}$. The two red dots represent the two experimental localizations as estimated in section 3.3.

\begin{tabular}{|l|c|c|c|}
\hline & $\begin{array}{c}\text { Ground truth localization } \\
\text { (no error) }\end{array}$ & $\begin{array}{c}\text { MUSIC localization } \\
(\simeq 2 \mathrm{~mm} \text { error })\end{array}$ & $\begin{array}{c}\text { Ground truth localization } \\
+6 \mathrm{~mm} \text { additional error }\end{array}$ \\
\hline Shape error Object 1 & $8.7 \%$ & $10.3 \%$ & $\begin{array}{c}13.7 \% \\
\text { (average over 50 points) }\end{array}$ \\
\hline Shape error Object 4 & $17.9 \%$ & $18.7 \%$ & $\begin{array}{c}35.1 \% \\
\text { (average over 50 points) }\end{array}$ \\
\hline
\end{tabular}

Table 3. Shape errors examples with different localization errors for a conductive (AR 2, $-30^{\circ}, 60 \mathrm{~mm}$ ) and an insulating ellipse (AR 1.5, 90 $\left.{ }^{\circ}, 60 \mathrm{~mm}\right)$.

the numerical and experimental results have two major origins. One is related to unavoidable experimental defects, the second is due to the approximation introduced by the model of objects on which the estimator is based. The former effects can be observed in the plots of axial and lateral currents in figures 5 and 6, which are representative of all our observations. They are due to the unavoidable noise on the emitted and measured signals, the geometric errors introduced by the gantry and the mechanical devices carrying the probe and supporting the objects, or time and space 
variations of the conductivity while performing experiments. In this regard, note that the analytical model (on which the estimation algorithm is based), needs to be informed by a constant conductivity which is measured at the beginning of the experiment with a conductivity meter (WTW Cond 197i) which provides a measurement with of about $0.5 \%$ precision. Beyond these experimental flaws, for any given object volume, the experimental results show the following trends:

- Trend 1: The shape estimation is better for conductors than insulators;

- Trend 2: The inclination angle estimation is fragile;

- Trend 3: The shape estimation performance decreases when the object aspect ratio increases and the probe gets closer to the object.

Trend 1, which is also observed on BEM simulations (see figure 16), can be explained by the fact that a conductive ellipsoid produces an electric response with an amplitude around twice that of an insulating object of same geometry. This basic fact of electrokinetics was first observed for spherical objects and then for ellipsoids in Rasnow (1996). In our experimental context, it implies that the signal/noise ratio of measurements is higher for conductive objects than insulating ones.

Trend 2 is due to two different reasons. First, when the aspect ratio increases, the quadripolar response is more and more excited and generates an apparent dipole rotated with respect to that of the expected dipolar response. As a result, the estimator, which is only based on a dipolar model of the response, interprets the object as an ellipsoid with axes shifted from their real to their apparent inclination. In short, the unmodeled quadripolar response strongly perturbs the dipolar model-based angle estimation. This is confirmed by remarking that for high aspect ratio, the error on angle estimation is maximal when the ellipsoidal object's major axis is perpendicular to the probe's axis. Referring to figure 11, in this pathological case, the sub-dipole parallel to the probe axis and closest to it, is strongly excited and the estimator interprets the object as an ellipsoid with a major axis aligned with the probe axis, i.e., the error on $\theta_{o}$ reaches $\pi / 2$. This phenomenon increases with the aspect ratio. On the other hand, when the aspect ratio decreases, referring to (14), the angle calculation is less and less well conditioned and the performance of the angle estimation drops too. In the limit case, where it is equal to 1 , the object is spherical and the calculation of $\theta_{o}$ is fully undetermined.

Trend 3 can be explained by the flaws of the model of the object response. In fact, as evoked in section 3.3, the electric response $\mathbf{E}_{1}=-\nabla \phi_{1}$ of an object immersed in a basal field $\mathbf{E}_{0}=-\nabla \phi_{0}$, is governed by an infinite multipolar expansion of the general form Ammari, Garnier, Kang, Lim and Yu (2014):

$$
\phi_{1}(\mathbf{r})=\frac{1}{4 \pi} \sum_{|\alpha|,|\beta|=1}^{+\infty} \frac{(-1)^{|\beta|}}{\alpha ! \beta !}\left(\partial^{\alpha} \frac{1}{r}\right) P_{\alpha \beta}\left(\partial^{\beta} \phi_{0}\right)(\mathbf{0}),
$$

where we used standard multi-index notations in $\mathbb{R}^{3}$ as well as $r=\|\mathbf{r}\|$, with $\mathbf{r}=x^{i} \mathbf{b}_{i}$ the position vector (in the object frame) at which the reflected potential field $\phi_{1}$ is evaluated. In this expansion $P_{\alpha \beta}$ defines the generalized polarization 
tensors (GPT) which model the multipolar momentums of the distribution of electric charges on the object boundaries due to its polarization by the basal field $\mathbf{E}_{0}(\mathbf{r})=\mathbf{E}_{0}(\mathbf{0})+\nabla \mathbf{E}_{0}(\mathbf{0}) . \mathbf{r}+\ldots$. In detail, $P_{11}$ is the matrix of the components of the leading order GPT denoted $\mathbf{P}$ in section 2.2, i.e. $P_{11}=P$. This tensor models the dipolar response of the object immersed in an uniform field, while $P_{2 \beta}$ models its quadripolar response in the same conditions. Now, let us remark that after the leading order contribution, the non-uniformity of the basal field excites the further GPT defined by $P_{12}$, while when the sensor gets closer to the object the quadripolar response $P_{21}$ of magnitude $\sim 1 / r^{3}$ can no longer be neglected in the model of measurements. This explains why the model on which the shape estimator is based, is more and more justified when the object size decreases with respect to the probe basal field, and as it gets further from the probe, a basic fact confirmed in the ideal numerical conditions of Lanneau et al. (2016). In the present experimental context we were obliged to consider large objects and to get the probe closer to them, as has already been remarked in section 3.3, to keep a signal/noise ratio compatible with the exploitation of the measurements. In these conditions, these further contributions become non-negligible in the total electric response (16). In particular, for a given volume, when the aspect ratio increases, the basal field lying along its domain is less and less uniform, and the $P_{12}$-terms of (16) become no longer negligible. This probably explains why the performance of shape estimation, which is based on the object dipolar response model $P_{11}$, falls when the aspect ratio increases. This interpretation is reinforced by a further observation. When the object main axis is perpendicular to the probe, the shape estimation performance falls too (see figure 18 and 19). In this case, since the basal field has a stronger gradient in the lateral direction (see figure 1-right), it is strongly non-uniform, and the $P_{12}$-response of the object perturbs the shape estimator.

\subsection{Improved shape estimation strategy}

Based on the above discussion, one can infer an improved strategy for shape estimation. As remarked before, the estimation of the inclination angle is fragile which decreases the performance of the shape estimator. However, this difficulty can be circumvented by adding after the localization step, a further step ensuring the self alignment of the probe with one of the symmetry axis of the ellipsoidal object. This can be performed incrementally through a convergent sequence of discreet rigid displacements as in Bai et al. (2015), or continuously through simple reactive control laws such as those proposed in Boyer et al. (2013). More precisely, it suffices to apply the feedback control

$$
\left(\begin{array}{c}
V_{x} \\
V_{y} \\
\Omega_{z}
\end{array}\right)=\left(\begin{array}{c}
k_{1} \delta I_{\mathrm{ax}, 2} \\
k_{2} I_{\mathrm{lat}, 1} \\
k_{2} I_{\mathrm{lat}, 2}
\end{array}\right),
$$

and to choose a set of gains $k_{i=1,2,3}$ able to steer the sensor in a stable pose aligned with the minor axis of the ellipsoid. Once such a reactive phase achieved, the inclination angle of the sensor is known to be zero, and one can control the sensor in open loop in order it joins a desired straight path with a desired $\theta_{o}$ and a desired lateral distance 

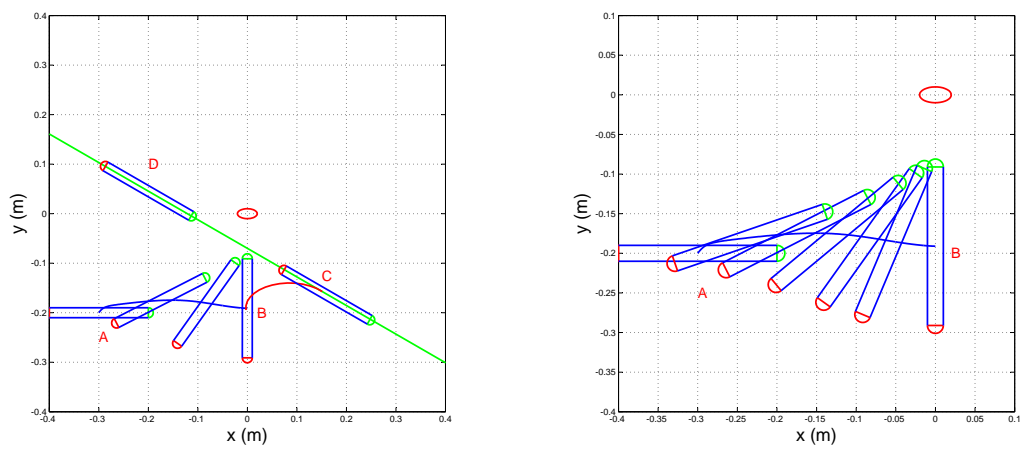

Figure 21. Left: Simulated motion of the probe in the improved shape estimation strategy (the lines $A B, B C, C D$ indicate the path of the probe's center). The probe starts from a pose A. Thanks to the control law (17), it is steered to the pose B, in which its body is aligned with the minor axis of the ellipsoid. The angle $\theta_{o}$ is then known to be zero and the probe is steered to pose $\mathrm{C}$ from which it starts a straight-line path with a prescribed $\theta_{o}$, here equal to $-30^{\circ}$. Right: snapshots of the probe when it is steered from pose $A$ to pose $B$.
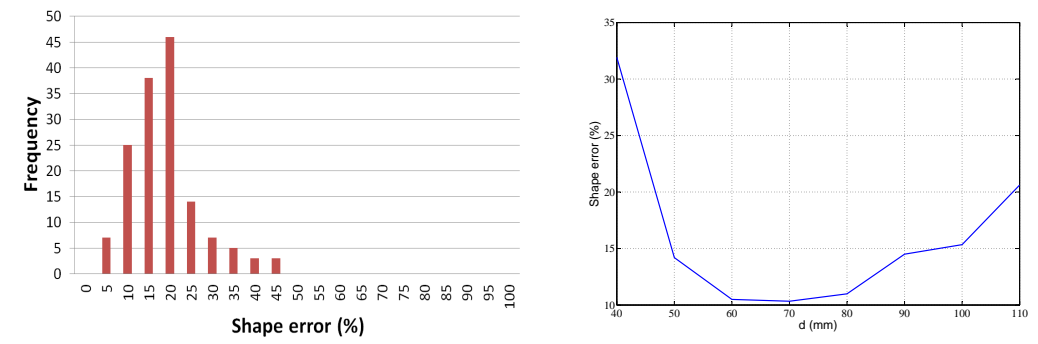

Figure 22. Left: Shape error distribution over the 137 experiments of table 2 when $\theta_{o}$ is a priori known. Right: Shape error vs lateral distance at $\theta_{o}=-30$.

$d$ (remind that the localization is known from the previous step). Such a reactive alignment is illustrated in figure 21 which shows a simulated path obtained with the law (17) when $k_{1}=1, k_{2}=10$ and $k_{3}=100$. Once the robot aligned, one can reapply the previous algorithm but with $\theta_{o}$ known. This improves the shape estimation as this is shown by figure 22-left, which displays the distribution of the shape estimation error obtained with the previous experiments, but with a priori knowledge of $\theta_{o}$. Figure 22right, shows that for an inclination angle $\theta_{o}=-30^{\circ}$ and a lateral distance $d=70 \mathrm{~mm}$, the shape estimation error drops to $10.3 \%$. This optimal configuration fully agrees with the above discussion (Trend 3) since in this case the object is at an optimal distance between noise and model validity criteria, while its major axis is close to be aligned with the basal field $\mathbf{E}_{0}$ so encouraging the object dipolar response on which the shape estimator is based. 


\section{Conclusion and perspectives for future research}

Based on a model of the measurements of a sensor providing the electric currents that flow across a set of electrodes arranged along a slender body, we have experimentally implemented a new approach for shape estimation of ellipsoidal objects. Assessed on 140 experiments, when the angle between the probe and the ellipsoid major axis is unknown, the average shape error is around $16 \%$, with more than $82 \%$ of the shape errors lower than $25 \%$. Moreover, when the angle is known a-priori, the shape error drops to $10 \%$. The approach is based on physical models of the sensor and the objects' response. In its improved version, it is hierarchically structured in three stages. The first stage consists of the detection of the object while identifying its electric nature. The second consists of localizing the object. The third consists of estimating its shape. This hierarchical strategy tends to support some of the conjectures in biology von der Emde (2006). Following Ammari, Boulier, Garnier and Wang (2014), this strategy has been performed by feeding the MUSIC algorithm with a dipolar model of the object's response. In these conditions, the algorithm behaves as a dipole localizer. Due to the large size of our experimental objects with respect to the basal field, the MUSIC algorithm has been adapted to localize the center of four sub-dipoles located on the boundaries of the ellipsoid whose barycenter turned out to be a good estimation of the object's center position. To capture the response of the object in the basal field of the sensor we used the leading-order generalized polarization tensor known as Polya-Szego tensor, which allows the modeling of the dipolar response of an object in a uniform electric field of any direction. This model has been here applied to our elliptic objects for which this tensor has a closed analytical expression. However, it has a wider generality, since in fact any shaped object responds at the leading order as an equivalent ellipsoid. As a result, the estimation strategy presented in the article should identify the equivalent ellipsoid of the leading order response of any object.

In the near future, we will continue this development in two directions. First, using a feedback controller of the form (17), the localization of the ellipsoidal object will be replaced by a reactive self positioning of the sensor at a given fixed pose with respect to the object center, this pose being independent of the object size, electric nature and aspect ratio. Second, we will advance the model of the object response in order to capture the contributions produced by the non-uniformity of the basal field, starting with the component $P_{12}$. While the closed analytical form of these tensors cannot be derived, one can tabulate them numerically using BEM and invert their plots with respect to the geometric parameters. Because of the high dimension of these further tensors, we will exploit the symmetry properties of the objects and measurements to get a minimal set of independent parameters describing their shape.

In the longer term, we aim to address the issue of underwater object estimation by an autonomous robot. Our approach required only boundaries left-right symmetry, a condition usually respected by our autonomous underwater robots (AUVs). However, several issues remain to be addressed. Firstly, we will have to derive an analytical model of the sensor response to the reflected field from the object using a non-slender bodied AUV. Secondly, the above approach assumes that the motions of the vehicle are 
perfectly known, but this is not the case for an AUV. In this context, electric sense will need to be combined with further inertial measurements.

\section{Acknowledgment}

Supported by European Unions Horizon 2020 research and innovation program under grant agreement No 640967. Project subCULTron: submarine cultures perform long-term robotic exploration of unconventional environmental niches. http://www.subcultron.eu

\section{References}

Ammari, H., Boulier, T., Garnier, J. and Wang, H. (2014), 'Shape recognition and classification in electro-sensing', Proceedings of the National Academy of Science (PNAS) 111(32), 431444.

Ammari, H., Garnier, J., Kang, H., Lim, M. and Yu, S. (2014), 'Generalized polarization tensors for shape description', Numerische Mathematik 126(2), 199-224.

Ammari, H. and Kang, H. (2007), Polarization and moment tensors, with applications to inverse problems and effective medium theory, Springer-Verlag New York.

Bai, Y., Snyder, J. B., Peshkin, M. and MacIver, M. A. (2015), 'Finding and identifying simple objects underwater with active electrosense', The International Journal of Robotics Research .

Bai, Y., Snyder, J., Silverman, Y., Peshkin, M. and MacIver, M. A. (2012), Sensing capacitance of underwater objects in bio-inspired electrosense, in '2012 IEEE/RSJ International Conference on Intelligent Robots and Systems (IROS)', pp. 1467-1472.

Boyer, F., Gossiaux, P., Jawad, B., Lebastard, V. and Porez, M. (2012), 'Model for a sensor inspired by electric fish', IEEE Transactions on Robotics 28(2), 492-505.

Boyer, F., Lebastard, V., Chevallereau, C., Mintchev, S. and Stefanini, C. (2015), 'Underwater navigation based on passive electric sense: New perspectives for underwater docking', The International Journal of Robotics Research pp. 1228-1250.

Boyer, F., Lebastard, V., Chevallereau, C. and Servagent, N. (2013), 'Underwater reflex navigation in confined environment based on electric sense', Robotics, IEEE Transactions on 29(4), 945-956.

Caputi, A. A., Budelli, R., Grant, K. and Bell, C. C. (1998), 'The electric image in weakly electric fish: physical images of resistive objects in gnathonemus petersii.', Journal of Experimental Biology 201(14), 2115-28.

Devaney, A. J. (2000), 'Super-resolution processing of multi-static data using time reversal and music', Unpublished . http://ece.neu.edu/fac-ece/devaney/preprints/paper02n_00.pdf.

Hofmann, V., Sanguinetti-Scheck, J. I., Gomez-Sena, L. and Engelmann, J. (2013), 'From static electric images to electric flow: towards dynamic perceptual cues in active electroreception', Journal of Physiology-Paris 107(12”), 95 - 106.

Krim, H. and Viberg, M. (1996), 'Two decades of array signal processing research: the parametric approach', Signal Processing Magazine, IEEE 13(4), 67-94.

Landau, L. and Lifshitz, E. (1984), Electrodynamics of Continuous Media, second ed, Pergamon Press, Oxford.

Prepared using sagej.cls 
Lane, D. (2012), Persistent autonomy artificial intelligence or biomimesis?, in 'Autonomous Underwater Vehicles (AUV), 2012 IEEE/OES', pp. 1-8.

Lanneau, S., Lebastard, V. and Boyer, F. (2016), Object shape recognition using electric sense and ellipsoid's polarization tensor, in 'In Proceedings of the IEEE International Conference on Robotics and Automation (ICRA)', pp. 4692-4699.

Lebastard, V., Chevallereau, C., Girin, A., Servagent, N., Gossiaux, P.-B. and Boyer, F. (2013), 'Environment reconstruction and navigation with electric sense based on a kalman filter', The International Journal of Robotics Research 32(2), 172-188.

Lissmann, H. W. and Machin, K. E. (1958), 'The mechanism of object location in Gymnarchus Niloticus and similar fish', Journal of Experimental Biology 35(2), 451-486.

Ljung, L. (1999), System identification (2nd ed.): theory for the user, Prentice Hall PTR.

Rasmussen, C. E. and Williams, C. K. I. (2006), Gaussian Processes for Machine Learning, the MIT Press.

Rasnow, B. (1996), 'The effects of simple objects on the electric field of Apteronotus', Journal of Comparative Physiology A 178(3), 397-411.

Schmidt, R. (1986), 'Multiple emitter location and signal parameter estimation', IEEE Transactions on Antennas and Propagation 34, 276-280.

Servagent, N., Jawad, B., Bouvier, S., Boyer, F., Girin, A., Gomez, F., Lebastard, V., Stefanini, C. and Gossiaux, P.-B. (2013), 'Electrolocation sensors in conducting water bio-inspired by electric fish', Sensors Journal, IEEE 13(5), 1865-1882.

Solberg, J. R., Lynch, K. M. and MacIver, M. A. (2008), 'Active electrolocation for underwater target localization', The International Journal of Robotics Research 27(5), 529-548.

von der Emde, G. (2006), 'Non-visual environment imaging and object detection through active electrolocation in weakly electric fish', Journal of Computational Physiology A 192, 601 612.

von der Emde, G. and Schwarz, S. (2002), 'Imaging of objects through active electrolocation in gnathonemus petersii', Journal of Physiology-Paris 96(56), 431 - 444.

Prepared using sagej.cls 